\title{
Key Structural Motifs Balance Metal Binding and Oxidative Reactivity in a Heterobimetallic Mn/Fe Protein
}

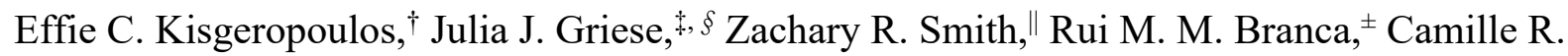
Schneider, ${ }^{\dagger}$ Martin Högbom, ${ }^{\star}$ and Hannah S. Shafaat ${ }^{*}, \|, \dagger$

"Department of Chemistry and Biochemistry and The Ohio State Biochemistry Program, The Ohio State University, $100 \mathrm{~W}$. 18th Ave., Columbus, OH, 43210, USA.

Department of Biochemistry and Biophysics, Stockholm University, SE-106 91 Stockholm, Sweden.

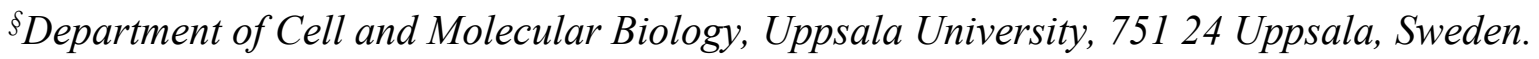

${ }^{ \pm}$Department of Oncology-Pathology, Science for Life Laboratory, Karolinska Institutet, SE-171 21 Solna, Sweden

\section{Supporting Information}




\section{Table of Contents}

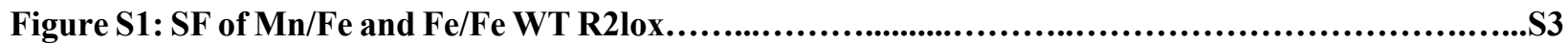

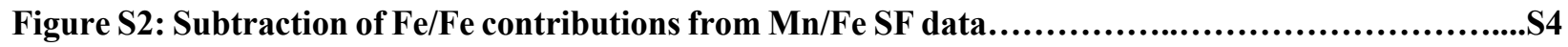

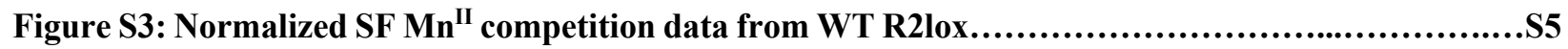

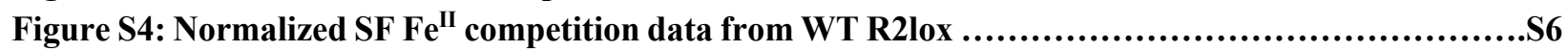

Figure S5: UV-vis kinetic traces of the WT, Y175F, and E69D R2lox resting states..........................S7

Figure S6: Representative spectral data from UV-vis competition experiment..........................S8

Figure S7: Representation of data analysis used to determine $t\left(A_{1 / 2}\right)$ values...............................S9

Figure S8: $\mathrm{Mn} / \mathrm{Fe}$ assembly at $390 \mathrm{~nm}$ monitored during SF metal competition experiments.............S10

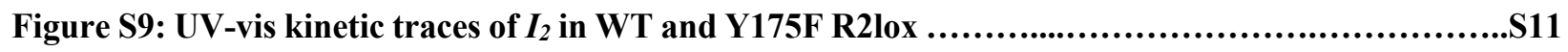

Figure S10: Kinetic traces monitoring the $I_{2}$ species at $390 \mathrm{~nm}$ in WT R2lox.........................S12

Figure S11: KinTek simulations of various metal binding scenarios for WT R2lox....................................S13

Figure S12: EPR of R2lox variants with only $\mathrm{Mn}^{\mathrm{II}}$ added at increasing concentrations...................S14

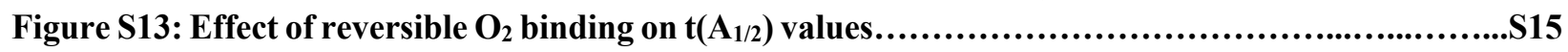

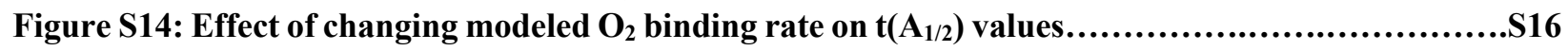

Figure S15: $\mathrm{Mn}^{\mathrm{II}}$ inhibition curves for various metal binding affinities.................................S17

Figure S16: Presence of $\mathrm{Fe}^{\mathrm{II}}$ in site $\mathrm{B}$ modulates $\mathrm{Mn}^{\mathrm{II}}$ binding to site A...............................S18

Figure S17: Effect of increased $k_{0 n} / k_{\text {off }}$ rates for both metals on $t\left(A_{1 / 2}\right)$ values..........................S19

Figure S18: Effect of increased $k_{\text {on }} / k_{\text {off }}$ rates for $\mathrm{Mn}^{\mathrm{II}}{ }_{\mathrm{A}}$ on $\mathrm{t}\left(\mathrm{A}_{1 / 2}\right)$ values...............................S20

Figure S19: Global fitting of KinTek models to the scaled experimental data..............................S21

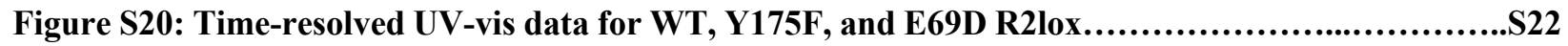

Figure S21: Comparison of UV-vis intermediate decay kinetics in WT and Y175F...................S23

Figure S22: SF of E69D Mn/Fe cofactor assembly monitored at relevant wavelengths....................S24

Figure S23: Effect of modulating both $I_{2}$ formation and $K_{\mathrm{FeB}}$ on inhibition curves.........................S25

Figure S24: Effect of modulating both $I_{2}$ decay and $K_{M n A}$ on inhibition curves..........................S26

Figure S25: Effect of modulating $I_{2}$ formation/decay rates on $\mathrm{Mn}^{\mathrm{II}}$ inhibition curves.......................S27

Figure S26: Crystal structure of the E69D-R2lox dimer in the reduced state............................S28

Figure S27: Mass spectrometry analysis of cross-link formation in Fe/Fe R2lox.........................S29

Figure S28: Cyclic voltammograms of WT R2lox at pH 6.0....................................................................330

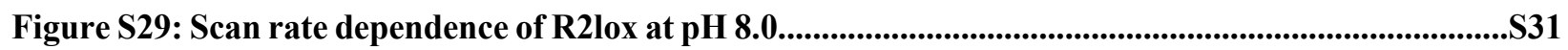

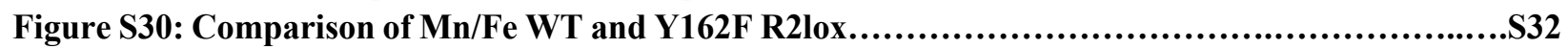

Figure S31: Gel showing purification of Y175F and E69D R2lox in relation to WT......................S33

Figure S32: Mn ${ }^{\mathrm{II}}$ inhibition curves from UV-vis overlaid with SF data...................................S34

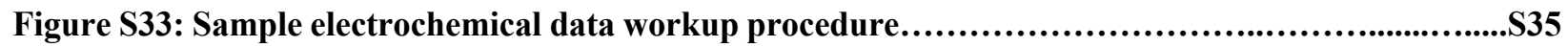

Table S1: Spin Hamiltonian parameters from EPR simulations of the R2lox resting state..................S36

Table S2: Efficiency of R2lox variants at $\mathrm{Mn}^{\mathrm{III}}(\mu-\mathrm{OH}) \mathrm{Fe} \mathrm{III}^{\mathrm{III}}$ cofactor and cross-link formation..............S37

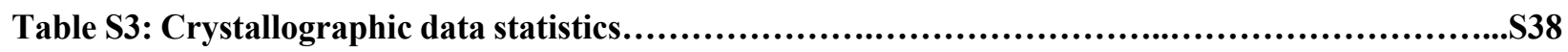

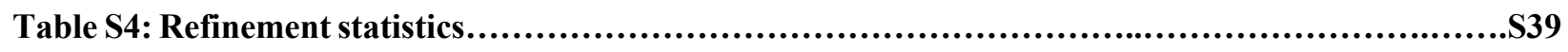

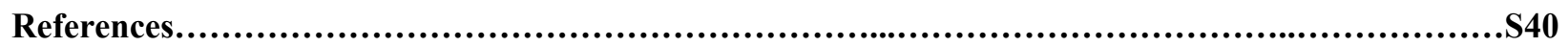



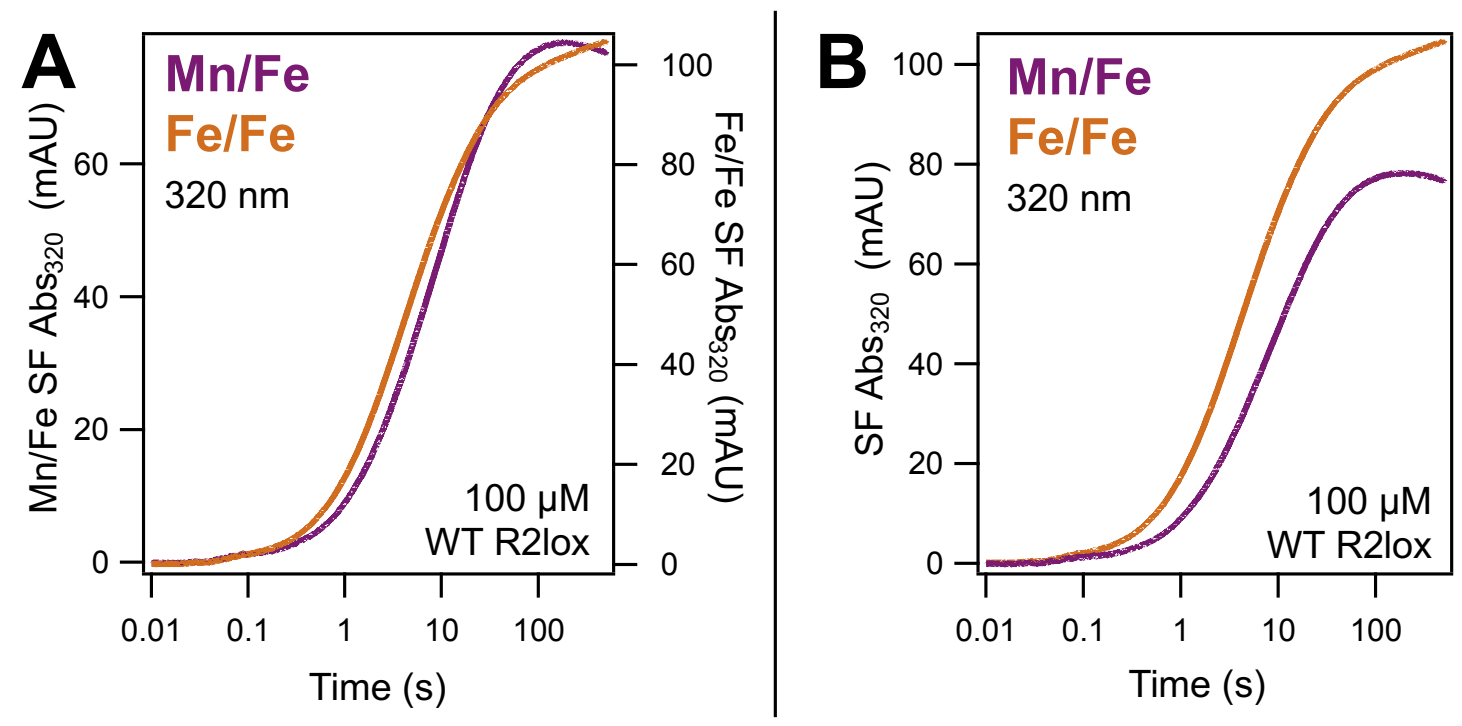

Figure S1. Normalized (A) or absolute (B) SF traces at $320 \mathrm{~nm}$ after mixing $100 \mu \mathrm{M}$ apo-R2lox with $100 \mu \mathrm{M} \mathrm{Mn}{ }^{\mathrm{II}}+100 \mu \mathrm{M} \mathrm{Fe} \mathrm{II}^{\mathrm{II}}$ (purple) or $200 \mu \mathrm{M} \mathrm{Fe}^{\mathrm{II}}$ (orange). While a noticeable yet small difference between the two cofactors is observed when traces are normalized to the maximum absorbance values, we note that the faster rate of assembly seen in Fe/Fe R2lox appears more pronounced in the absolute SF traces. Data represent the average of 4 biochemical replicates with 1-4 technical replicates each. 


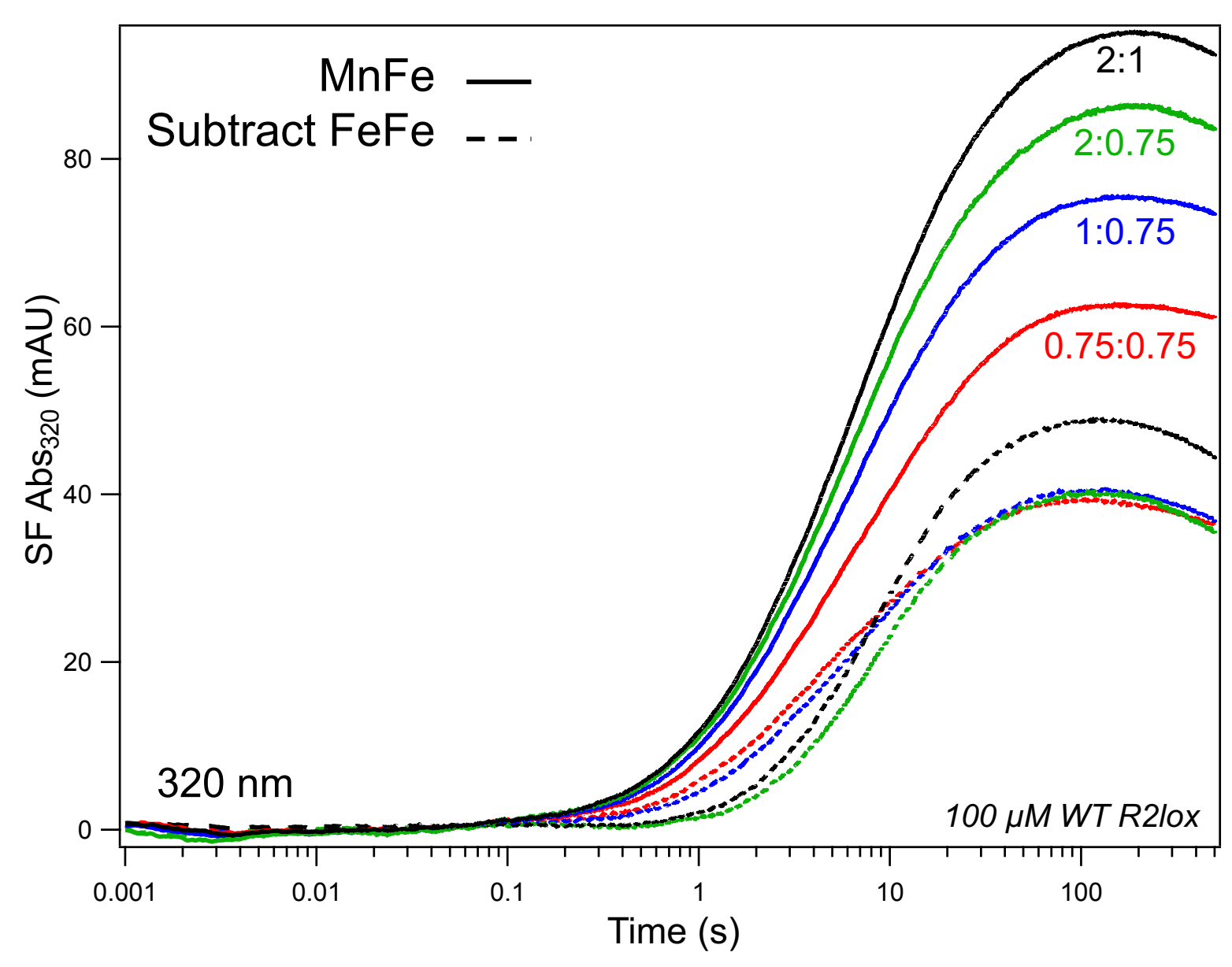

Figure S2. Results of subtracting $\mathrm{Fe} / \mathrm{Fe}$ R2lox stopped-flow data from $\mathrm{Mn} / \mathrm{Fe}$ in an attempt to resolve contributions from only the $\mathrm{Mn} / \mathrm{Fe}$ cofactor formation. For equivalently fast binding of $\mathrm{Fe}^{\mathrm{II}}$ to either site in either cofactor (and greater rates of $\mathrm{Fe}^{\mathrm{II}}$ binding than $\mathrm{Mn}^{\mathrm{II}}$ ), approximately $2 / 3$ of the $\mathrm{Fe}^{\mathrm{II}}$ in solution would be utilized in the formation of $\mathrm{Fe} / \mathrm{Fe}$ cofactors, resulting in $1 / 3$ of the total protein metalating as $\mathrm{Fe} / \mathrm{Fe}$ R2lox. The remaining $1 / 3$ of $\mathrm{Fe}^{\mathrm{II}}$ would be utilized in $1: 1$ binding with $\mathrm{Mn}^{\mathrm{II}}$ to give $1 / 3$ of total protein metalating as $\mathrm{Mn} / \mathrm{Fe}$ R2lox, leaving $1 / 3$ of the protein in its apo form. For a dataset of approximately equivalent traces, i.e. same total metal concentration across $\mathrm{Mn} / \mathrm{Fe}$ and $\mathrm{Fe} / \mathrm{Fe}$ data, the $\mathrm{Fe} / \mathrm{Fe}$ intensity was multiplied by a factor of $1 / 3$ and subtracted from that of the $\mathrm{Mn} / \mathrm{Fe}$. Were only kinetics playing a role, we would expect to be able to replicate the data prior to subtraction with less intensity and delayed onset. The reverse order of the appearance of the $0.75: 0.75$ and 1:0.75 traces after subtraction indicate additional levels of control (e.g., thermodynamics) are at play. 


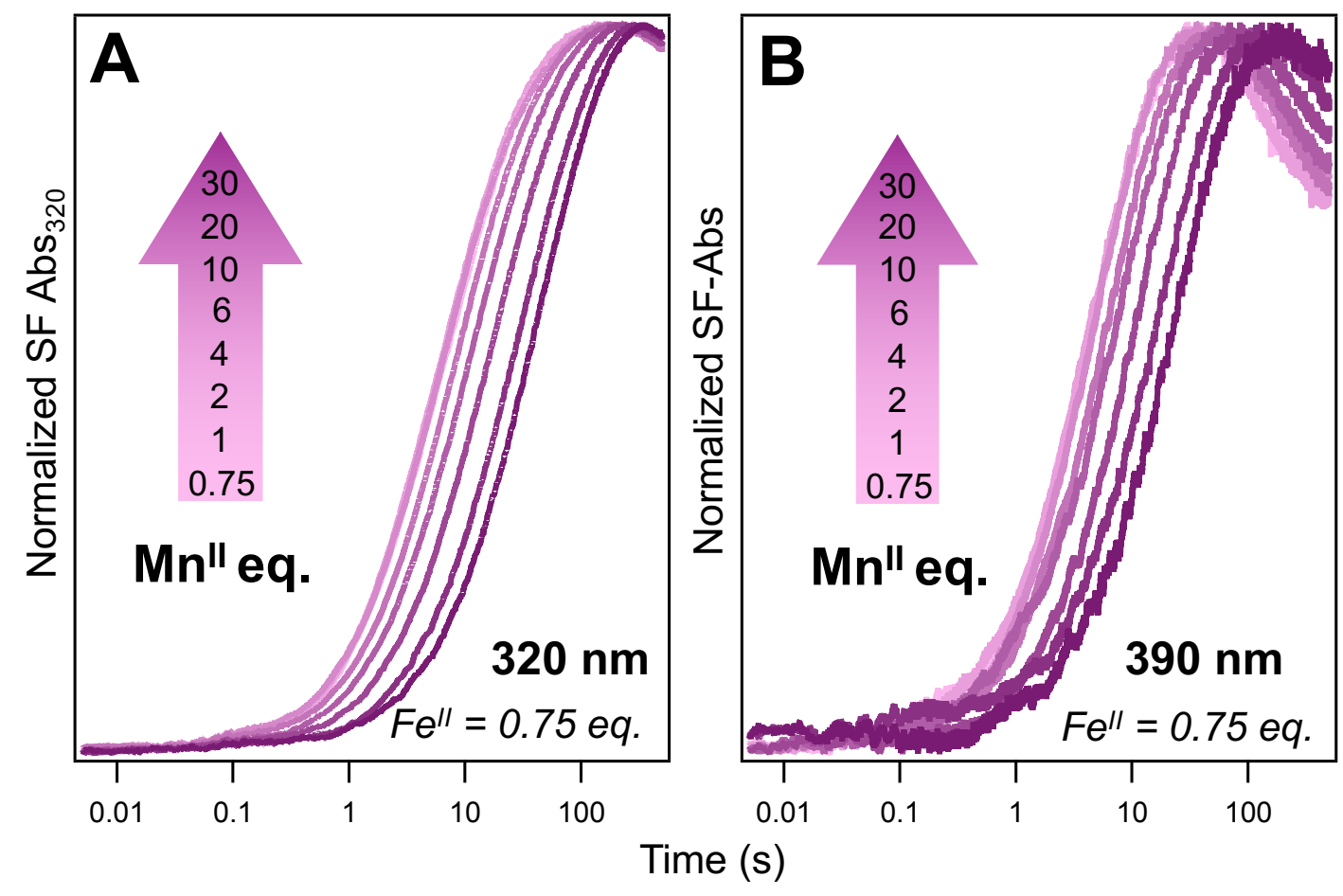

Figure S3. Normalized SF absorption kinetic traces data for $\mathrm{Mn}^{\mathrm{II}}$ competition experiments on WT R2lox monitoring (A) the resting state at $320 \mathrm{~nm}$ and (B) intermediate $I_{2}$ formation at $390 \mathrm{~nm}$. Increasing $\mathrm{Mn}^{\mathrm{II}}$ results in a pronounced delay of peak formation at both wavelengths. 

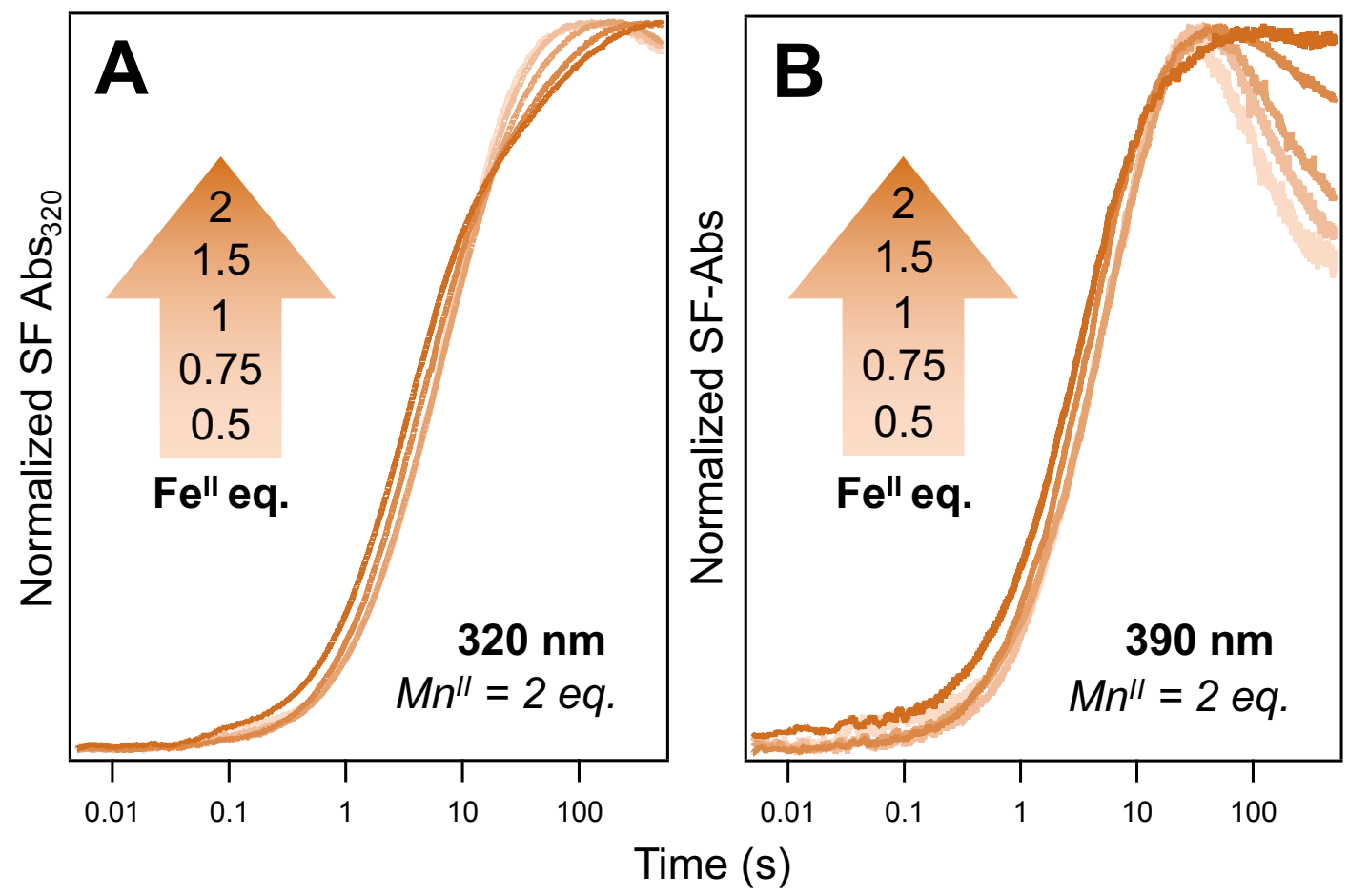

Figure S4. Normalized SF absorption kinetic traces data for $\mathrm{Fe}^{\mathrm{II}}$ competition experiments on WT R2lox monitoring (A) the resting state at $320 \mathrm{~nm}$ and (B) intermediate $I_{2}$ formation at $390 \mathrm{~nm}$. Increasing $\mathrm{Fe}^{\mathrm{II}}$ does not produce the same inhibitory effect on the rate of formation as seen with increasing $\mathrm{Mn}^{\mathrm{II}}$. The subtle increase in rates of formation observed at super-stoichiometric $\mathrm{Fe}^{\mathrm{II}}$ levels are attributed to the formation of $\mathrm{Fe} / \mathrm{Fe}$ cofactors. The change in shape of these traces at 320 $\mathrm{nm}$ and decreasing rate of decay for the $390 \mathrm{~nm}$ feature can also be attributed to $\mathrm{Fe} / \mathrm{Fe} \mathrm{R} 2 \mathrm{lox}$, which has a different optical profile than $\mathrm{Mn} / \mathrm{Fe}$ R2lox. 

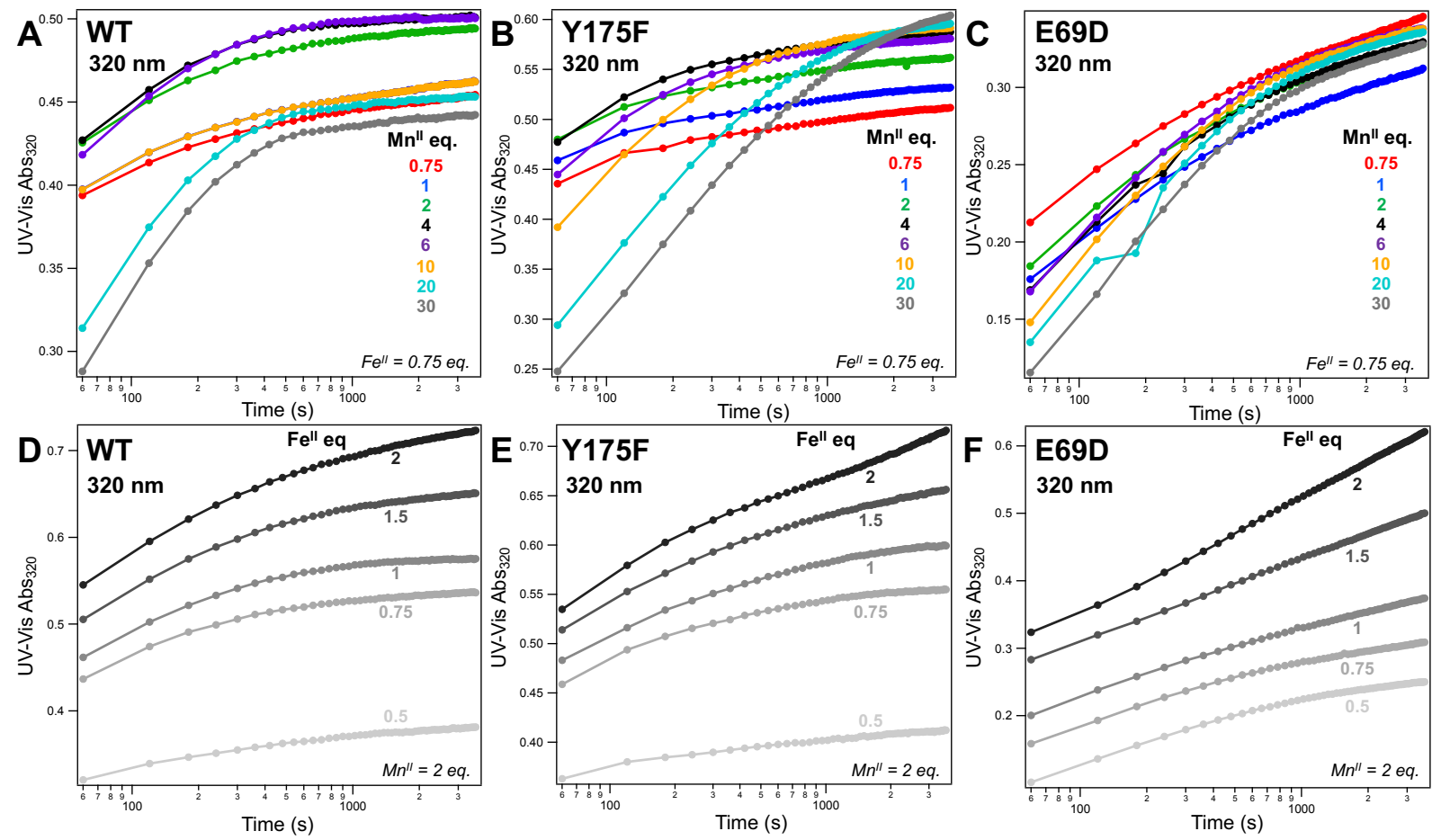

Figure S5. UV-vis kinetic traces monitoring the resting state formation at $320 \mathrm{~nm}$ in (A, D) WT, (B, E) Y175F, and (C, F) E69D R2lox as a function of increasing $\mathrm{Mn}^{\mathrm{II}}$ (top) or $\mathrm{Fe}^{\mathrm{II}}$ (bottom) concentration. Traces obtained from time-resolved UV-vis spectra collected from 1 to 60 min. 

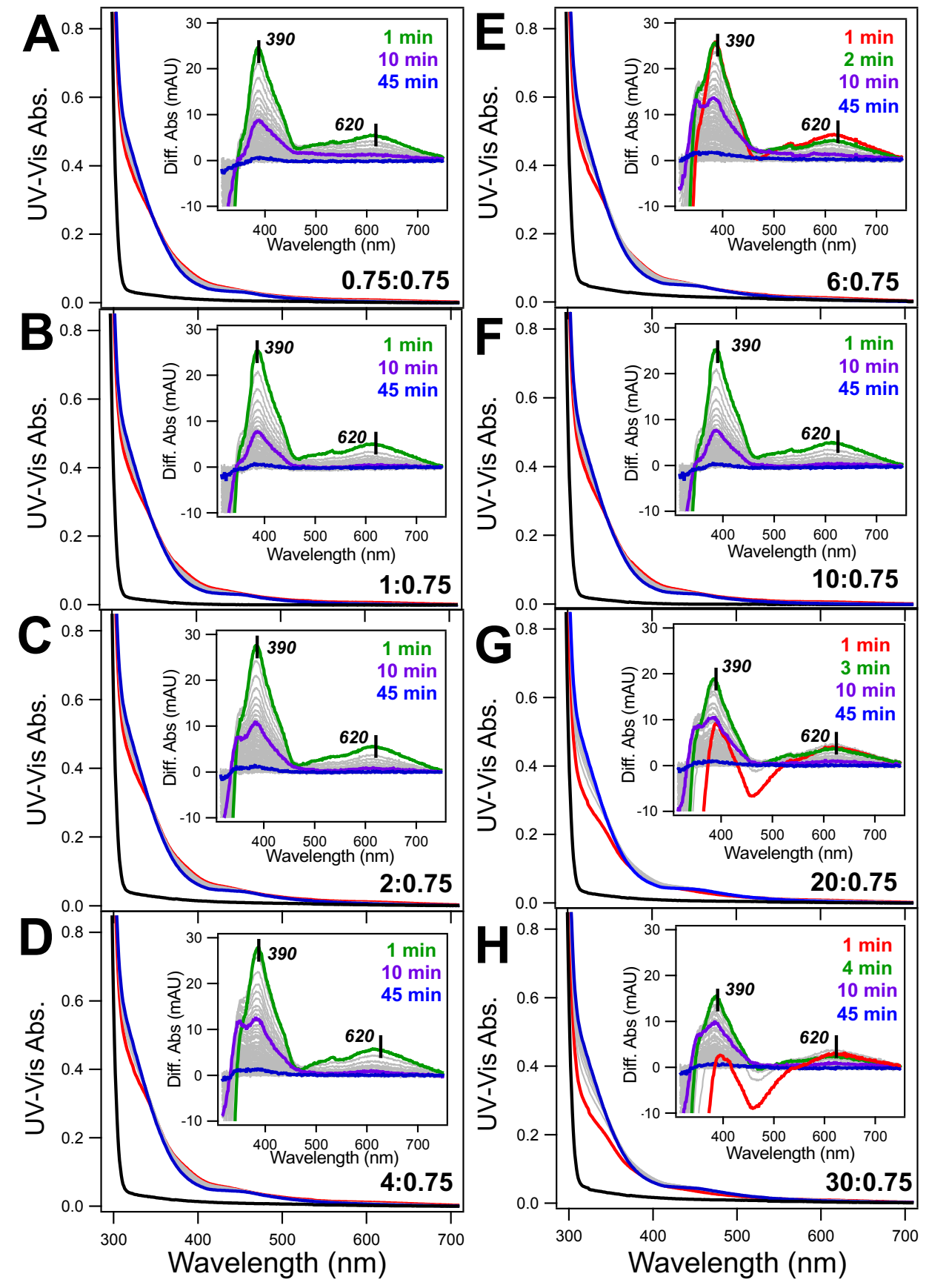

Figure S6. Representative spectral data from UV-vis competition experiments that vary the concentration of $\mathrm{Mn}^{\mathrm{II}}$. (A-H) Raw absorbance data are shown collected from 1-60 minutes, with the apo-R2lox (black), first minute ( $\mathrm{red}$ ), and final minute (blue), indicated. (A-H, insets) Corresponding difference spectra for each experiment, with the final trace subtracted from all previous traces. First minute (red) and/or maximum intensity at $390 \mathrm{~nm}$ (green), 10 minutes (purple) and 45 minutes (blue) are denoted on each. 


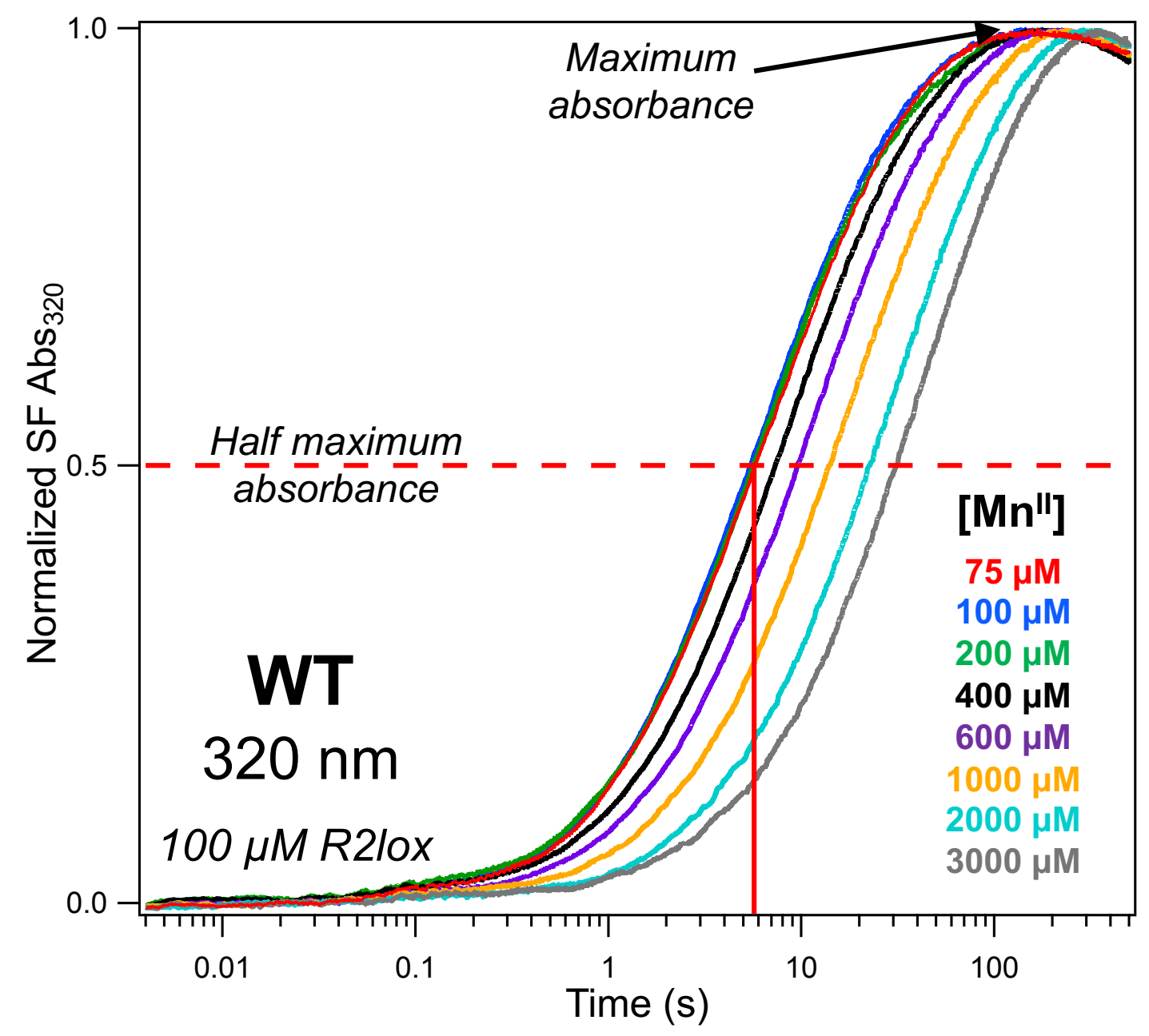

Figure S7. Representative graph showing data analysis procedure used to determine $t\left(A_{1 / 2}\right)$ values. Data traces were normalized, and a horizontal line drawn at half the maximum absorbance. The time at which each data trace crosses the half maximum absorbance line is recorded as the $t\left(\mathrm{~A}_{1 / 2}\right)$ for that datapoint. $\mathrm{X}$-axis value indicated by the red line shows the $\mathrm{t}\left(\mathrm{A}_{1 / 2}\right)$ when $75 \mu \mathrm{M} \mathrm{Mn} \mathrm{m}^{\mathrm{II}}$ is used. 


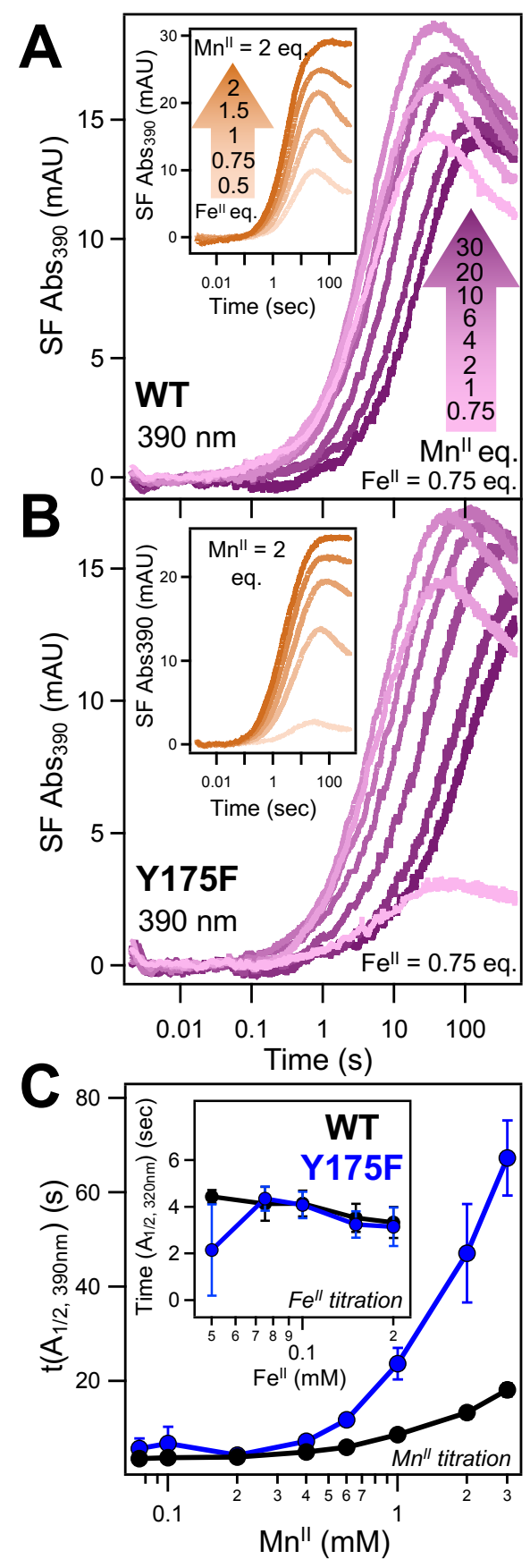

Figure S8. SF of Mn/Fe cofactor assembly in (A) WT and (B) Y175F R2lox as a function of metal concentration. $\mathrm{Fe}^{\mathrm{II}}$ held at 0.75 eq. of apo-R2lox and formation of $I_{2}$ at $390 \mathrm{~nm}$ monitored with increasing $\mathrm{Mn}^{\mathrm{II}}$. (C) Inhibition curves generated using experimental data at $390 \mathrm{~nm}$. Insets show corresponding $\mathrm{Fe}^{\mathrm{II}}$ competition data and $\mathrm{Fe}^{\mathrm{II}}$ inhibition curves. 

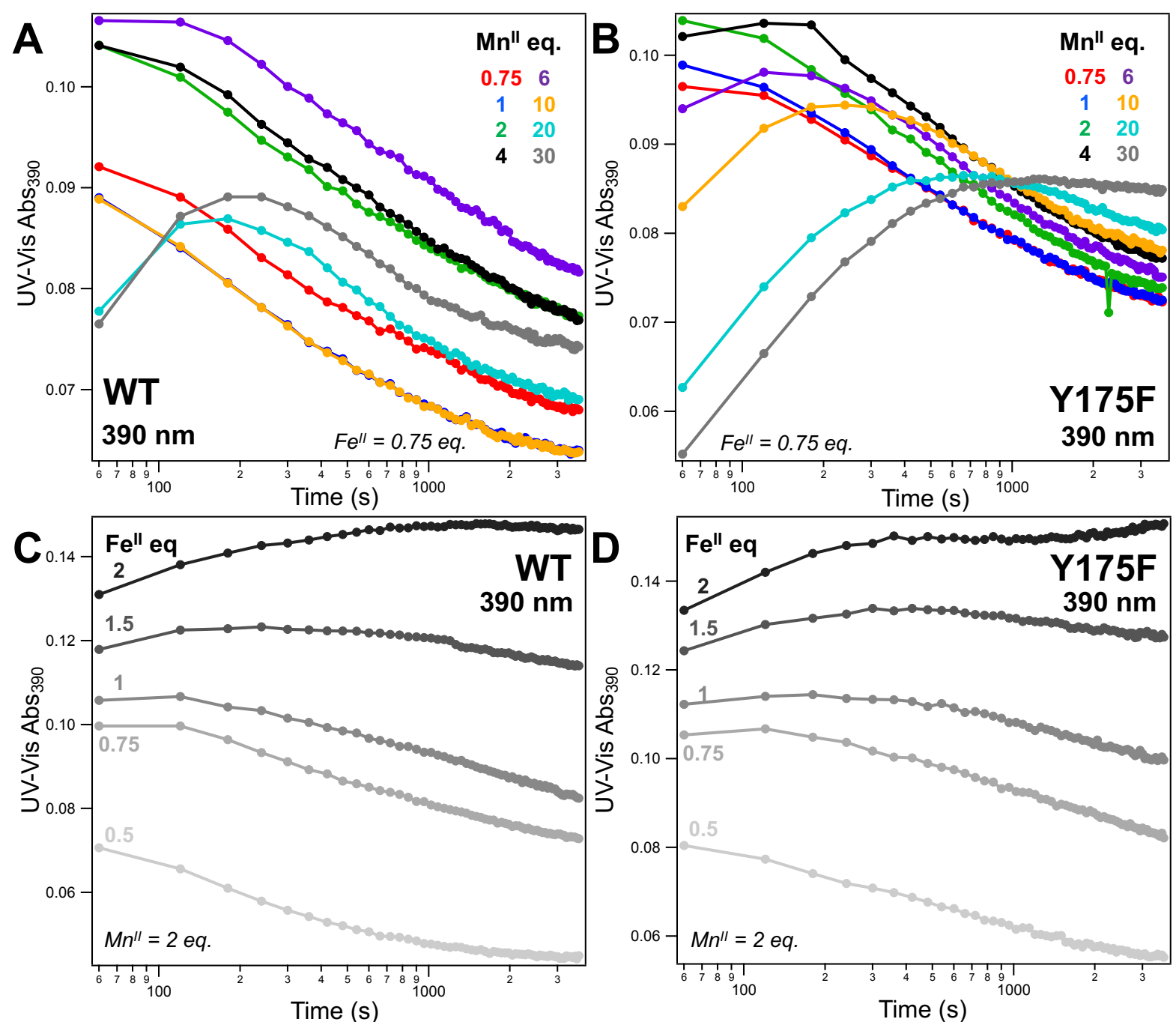

Figure S9. UV-vis kinetic traces of $I_{2}$ formation at $390 \mathrm{~nm}$ in (A, C) WT and (B, D) Y175F R2lox as a function of increasing $\mathrm{Mn}^{\mathrm{II}}$ (top) or $\mathrm{Fe}^{\mathrm{II}}$ (bottom) concentration. Traces obtained from timeresolved UV-vis spectra collected from 1 to $60 \mathrm{~min}$. As $\mathrm{Mn}^{\mathrm{II}}$ concentration increases, the formation of $I_{2}$ becomes well-resolved in the UV-vis kinetic traces, matching the delayed peak formation observed in the SF data, while the $390 \mathrm{~nm}$ features for both WT and Y175F exhibit the same response to increasing $\mathrm{Fe}^{\mathrm{II}}$ in the UV-vis compared to SF data (Figure S8). 


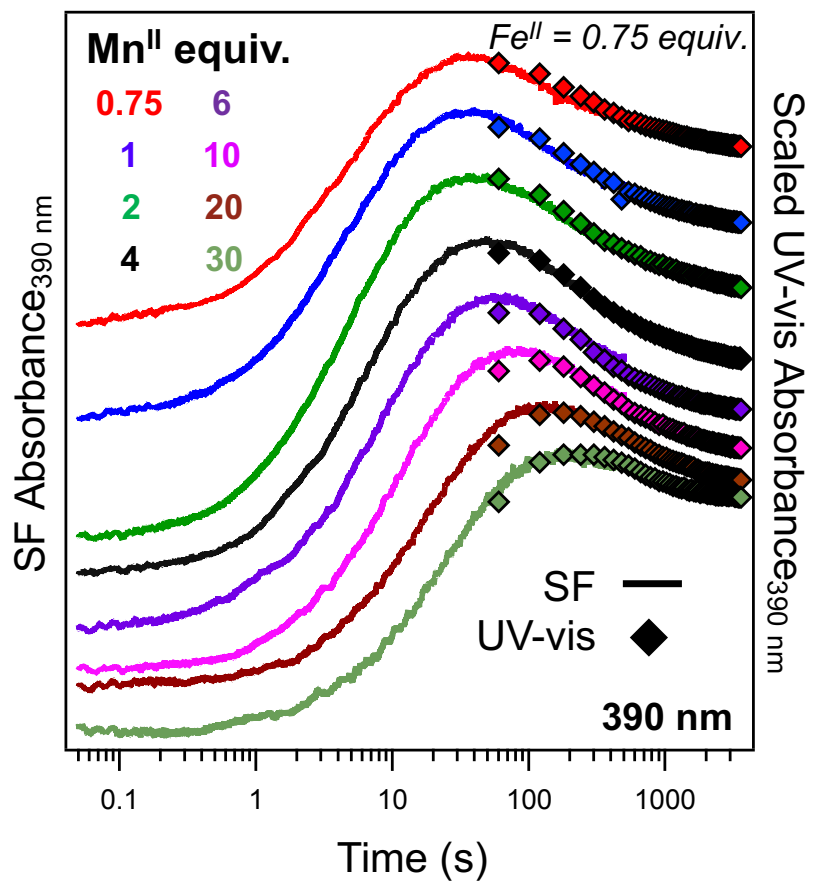

Figure S10. Kinetic traces monitoring the $I_{2}$ species at $390 \mathrm{~nm}$ in WT R2lox as a function of increasing $\mathrm{Mn}^{\mathrm{II}}$. Kinetic traces were obtained for $I_{2}$ formation and decay using either SF (lines) or UV-vis (diamonds) absorption data. The same experiment was performed using both techniques, and the resulting UV-vis data set was scaled and overlaid to showcase the good agreement with the SF absorbance kinetics. 

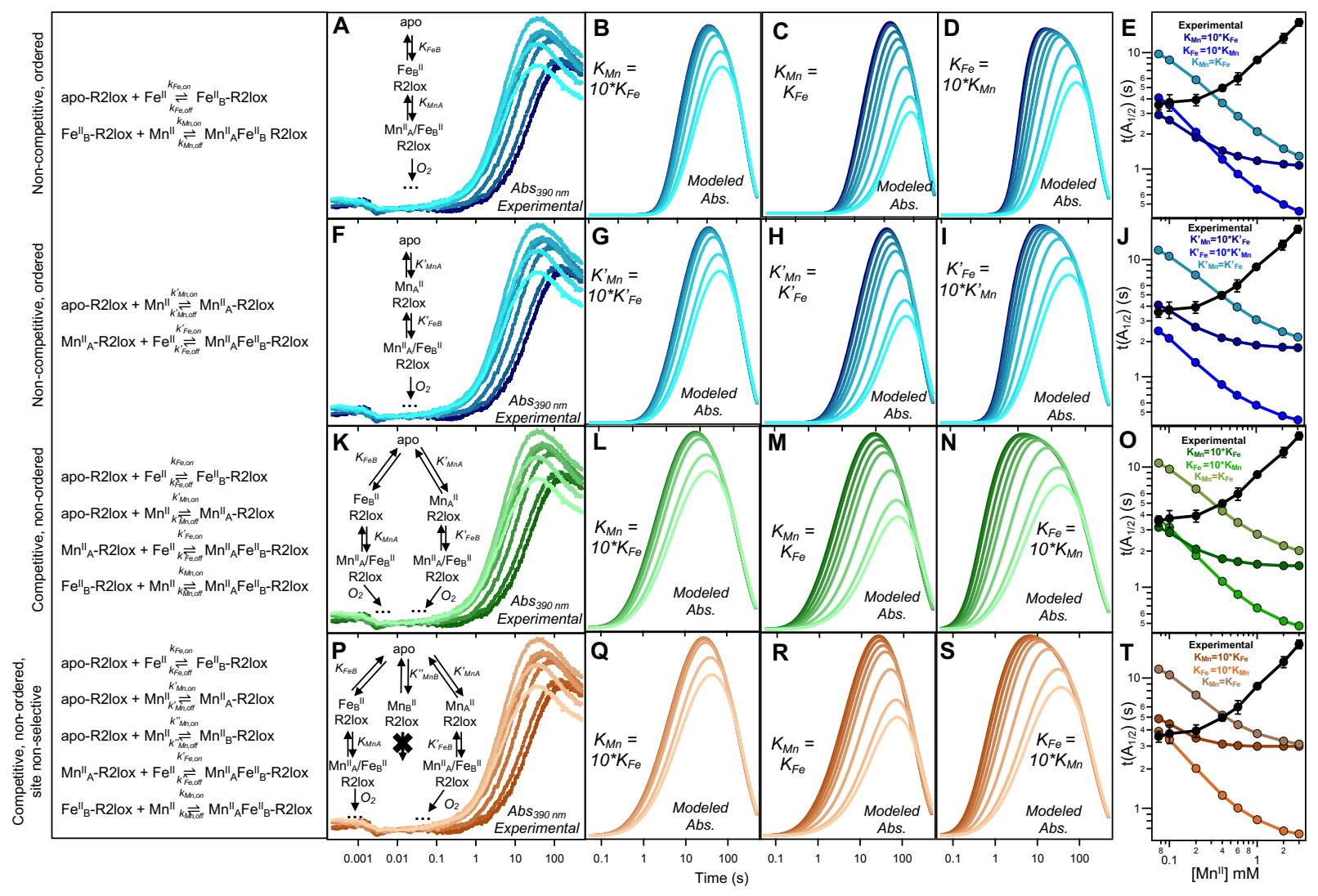

Figure S11. $I_{2}$ formation during SF $\mathrm{Mn}^{\mathrm{II}}$ competition experiments in WT R2lox is simulated using various metal binding models and relative metal binding affinities. On the left are the fundamental reactions for each model, from initial metal binding through formation of the $\mathrm{Mn}^{\mathrm{II}}{ }_{\mathrm{AFe}} \mathrm{II}_{\mathrm{B}}-\mathrm{R} 2 \mathrm{lox}$ core, corresponding to Eqs. 1 - 3 of the competitive/ordered model shown in the Experimental Section; all subsequent steps (Eqs. 4 - 10) remained the same. Schemes for metal binding scenarios as labeled: (A - E) Non-competitive/ordered, with $\mathrm{Fe}^{\mathrm{II}}$ binding first in site $\mathrm{B}, \mathrm{Mn}^{\mathrm{II}}$ binding second in site $\mathrm{A}$, and subsequent $\mathrm{O}_{2}$ activation $(\mathbf{F}-\mathbf{J})$ non-competitive/ordered, with $\mathrm{Mn}^{\mathrm{II}}$ binding first in site $\mathrm{A}, \mathrm{Fe}^{\mathrm{II}}$ binding second to site $\mathrm{B}$, followed by productive $\mathrm{O}_{2}$ activation $(\mathbf{K}-\mathbf{O})$ competitive/non-ordered, with either metal able to bind first and support $\mathrm{O}_{2}$ activation, and (P T) competitive/non-ordered/site non-selective, in which either metal can bind first and $\mathrm{Mn}^{\mathrm{II}}$ is able to bind in site $\mathrm{B}$, but ultimately only the $\mathrm{Mn}^{\mathrm{II}} / \mathrm{Fe}^{\mathrm{II}}{ }_{\mathrm{B}}$ configuration is productive, and $\mathrm{Mn}^{\mathrm{II}}$ binding in site B prevents any metal from binding to site A. Schemes shown as insets to identical experimental data (shown in different color schemes for more facile comparison to the models) in panels $\mathbf{A}, \mathbf{F}, \mathbf{K}$, and $\mathbf{P}$. Three regimes modeled for each scenario: $K_{M n A}=10 * K_{F e B}(\mathbf{B}, \mathbf{G}, \mathbf{L}, \mathbf{Q})$, $K_{M n A}=K_{F e B}(\mathbf{C}, \mathbf{H}, \mathbf{M}, \mathbf{R})$, and $K_{F e B}=10^{*} K_{M n A}(\mathbf{D}, \mathbf{I} \mathbf{N}, \mathbf{S}) . \mathrm{Mn}^{\mathrm{II}}$ inhibition curves generated from each modeled scenario and compared to experimental data $(\mathbf{E}, \mathbf{J}, \mathbf{O}, \mathbf{T})$. For all models, $K_{M n A}=$ $K^{\prime}{ }_{M n A}=K^{\prime \prime}{ }_{M n B}$ and $K_{F e B}=K^{\prime}{ }_{F e B}$, with $K_{M n A}$ and $K_{F e B}=10^{3}-10^{4} \mathrm{M}^{-1}$; $\mathrm{k}_{\text {off }}$ for all metal binding was set to $10 \mathrm{~s}^{-1}$ and $\mathrm{k}_{\text {on }}$ was adjusted appropriately to give the indicated affinity. 

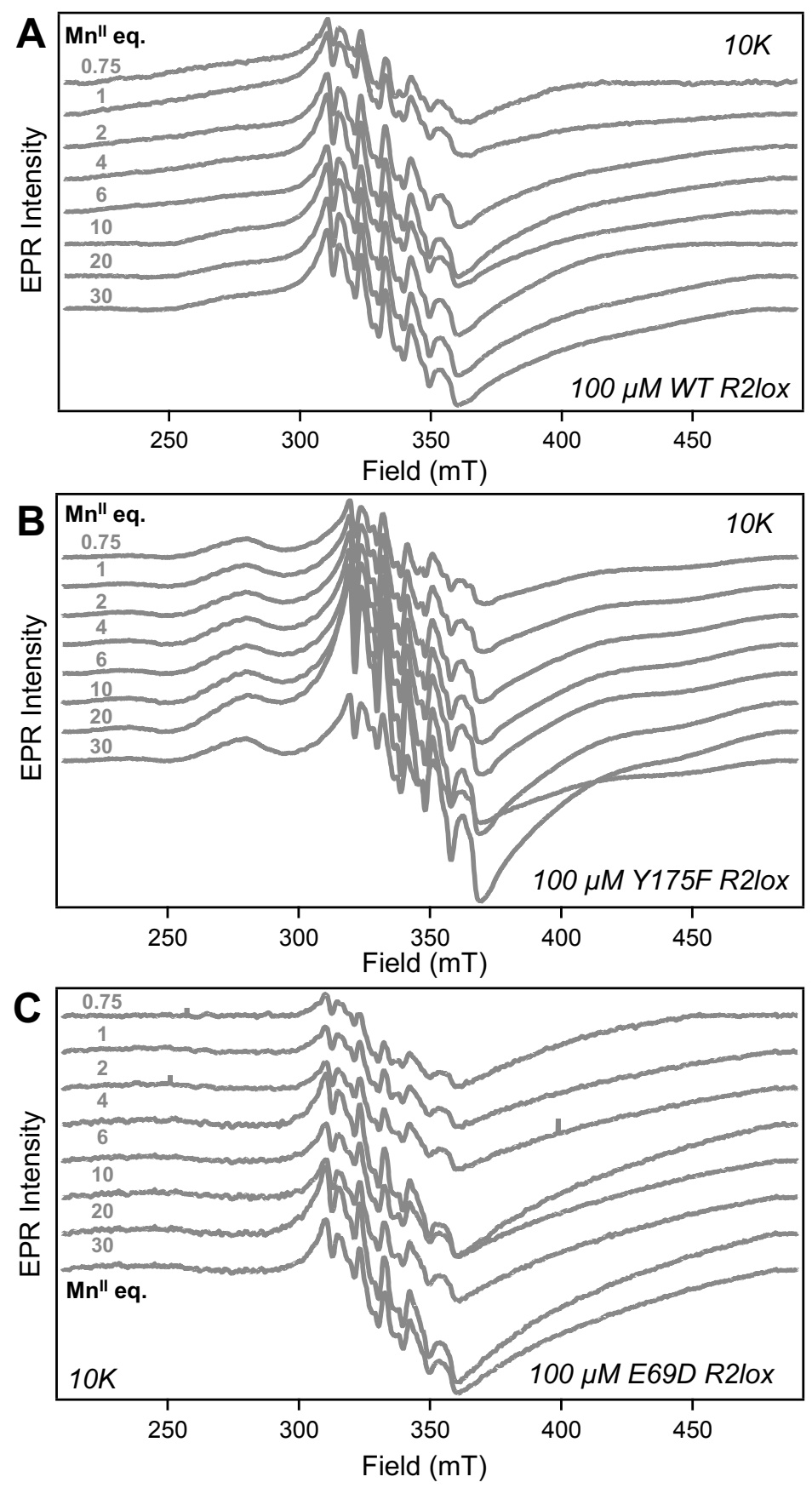

Figure S12. X-band CW EPR spectra of (A) WT, (B) Y175F, and (C) E69D R2lox treated with increasing $\left[\mathrm{Mn}^{\mathrm{II}}\right]$ and desalted prior to freezing. Characteristic $\mathrm{Mn}^{\mathrm{II}}$ signal is observed due to

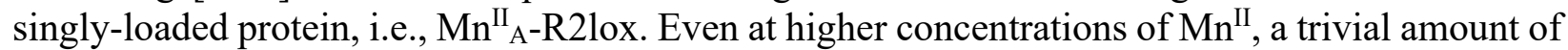
dimer signal due to $\mathrm{Mn}^{\mathrm{II}}{ }_{\mathrm{A}} / \mathrm{Mn}^{\mathrm{II}}$ is observed in WT and E69D; Y175F shows a more pronounced $\mathrm{Mn}_{2}{ }^{\mathrm{II}}$ dimer signal than WT R2lox, though it falls far short of that seen in the class Ic R2 proteins, ${ }^{1}$ which are known to readily form these dimers. 

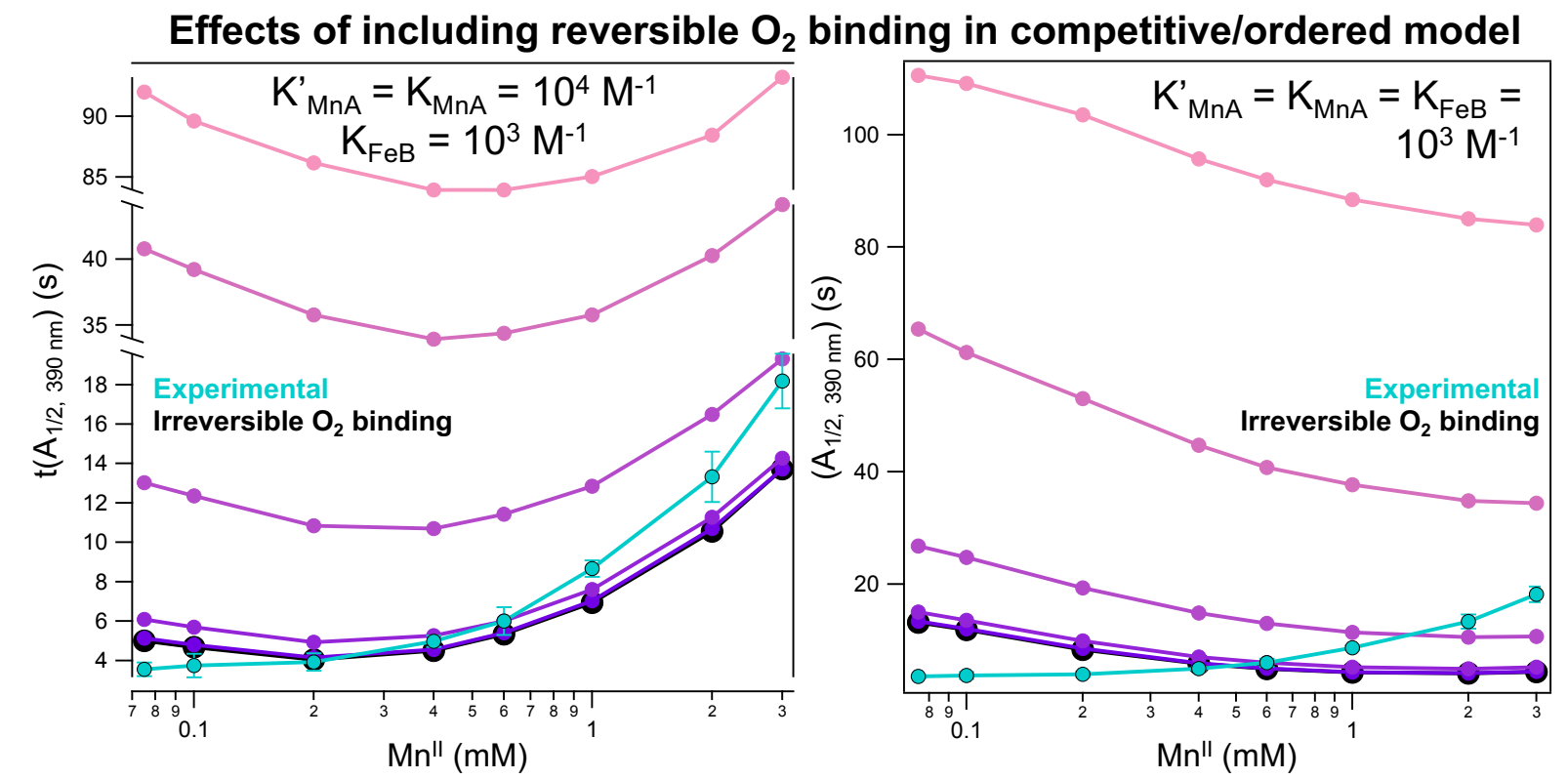

\begin{tabular}{|c|c|c|}
\hline $\mathbf{O}_{\mathbf{2}} \mathbf{k}_{\text {on }}\left(\mathbf{M}^{-\mathbf{1}} \mathbf{s}^{-1}\right)$ & $\mathbf{O}_{\mathbf{2}} \mathbf{k}_{\text {off }}\left(\mathbf{s}^{-1}\right)$ & $\left.\mathbf{K}_{\mathbf{0} 2} \mathbf{( M}^{-1}\right)$ \\
\hline $1 \times 10^{6}$ & 0 & - \\
\hline $1 \times 10^{6}$ & $1 \times 10^{2}$ & $1 \times 10^{4}$ \\
\hline $1 \times 10^{6}$ & $1 \times 10^{3}$ & $1 \times 10^{3}$ \\
\hline $1 \times 10^{6}$ & $1 \times 10^{4}$ & $1 \times 10^{2}$ \\
\hline $1 \times 10^{6}$ & $1 \times 10^{5}$ & $1 \times 10^{1}$ \\
\hline $1 \times 10^{6}$ & $1 \times 10^{6}$ & $1 \times 10^{0}$ \\
\hline
\end{tabular}

Figure S13. Effects of including reversible $\mathrm{O}_{2}$ binding on modeled $\mathrm{t}\left(\mathrm{A}_{1 / 2}\right)$ values using either the competitive/ordered model (left) or the competitive/ordered model with $\mathrm{Mn}^{\mathrm{II}} \mathrm{A}$ binding affinity $\left(\mathrm{K}_{\mathrm{MnA}}\right)$ equal to $\mathrm{Fe}^{\mathrm{II}}{ }_{\mathrm{B}}$ binding affinity $\left(\mathrm{K}_{\mathrm{FeB}}\right)$ (right). $\mathrm{O}_{2} \mathrm{k}_{\text {off }}$ rates were varied by factors of 10 until equaling $\mathrm{O}_{2} \mathrm{k}_{\text {on }}$. Each model without reversible $\mathrm{O}_{2}$ binding (black) and experimental data (teal) shown for comparison. In both situations, $\mathrm{t}\left(\mathrm{A}_{1 / 2}\right)$ values increase as $\mathrm{O}_{2}$ binding affinity decreases, but the trends observed with irreversible $\mathrm{O}_{2}$ binding remain the same. For all metal binding equilibrium constants, $\mathrm{k}_{\mathrm{off}}=10 \mathrm{~s}^{-1}$ and $\mathrm{k}_{\mathrm{on}}$ adjusted accordingly. 


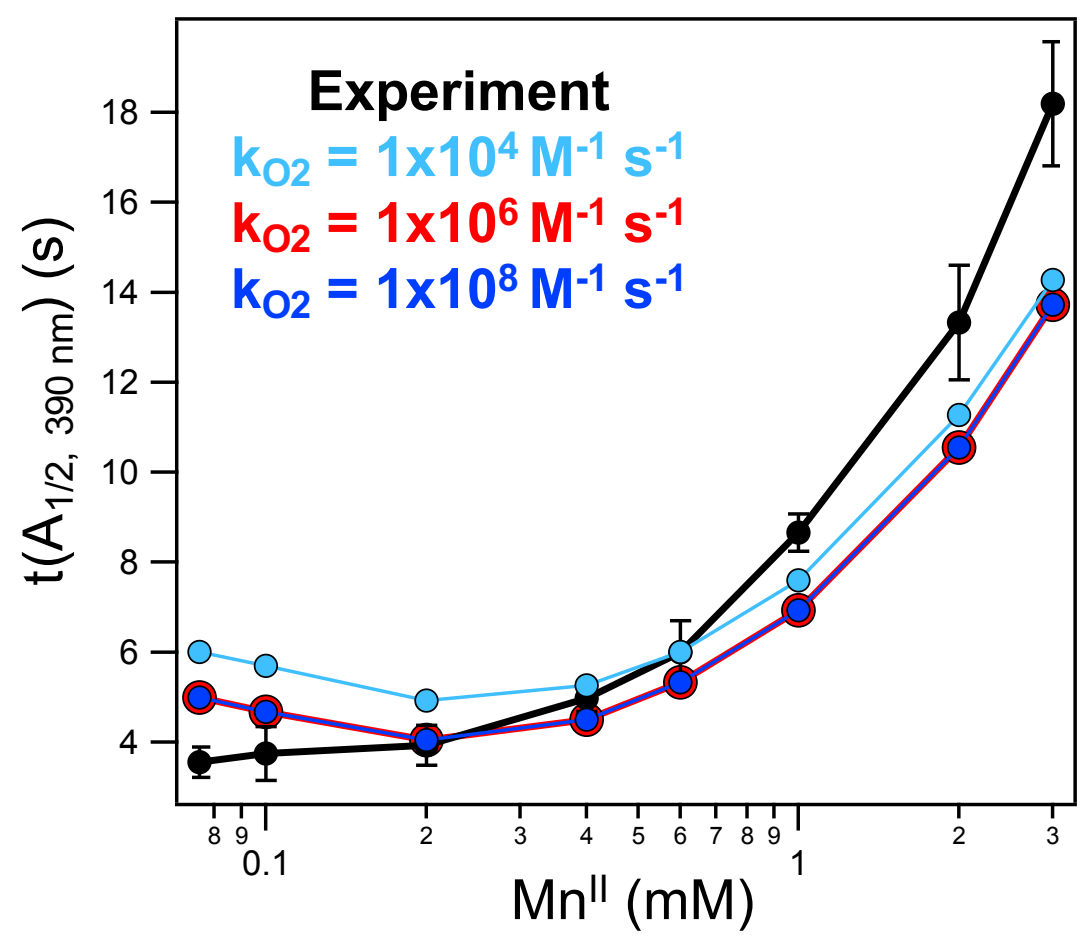

Figure S14. Effects of changing modeled $\mathrm{O}_{2}$ binding rate on modeled $t\left(\mathrm{~A}_{1 / 2}\right)$ values using the competitive/ordered model with metal binding equilibrium constants given in Figure $\mathrm{S} 13 \mathrm{~A}$. $\mathrm{O}_{2}$ binding was modeled to be two orders of magnitude slower (dark blue) or faster (light blue) than the value used in all other models $(\mathrm{red})$. No significant effect on the $t\left(\mathrm{~A}_{1 / 2}\right)$ values or inhibition trends were observed. 

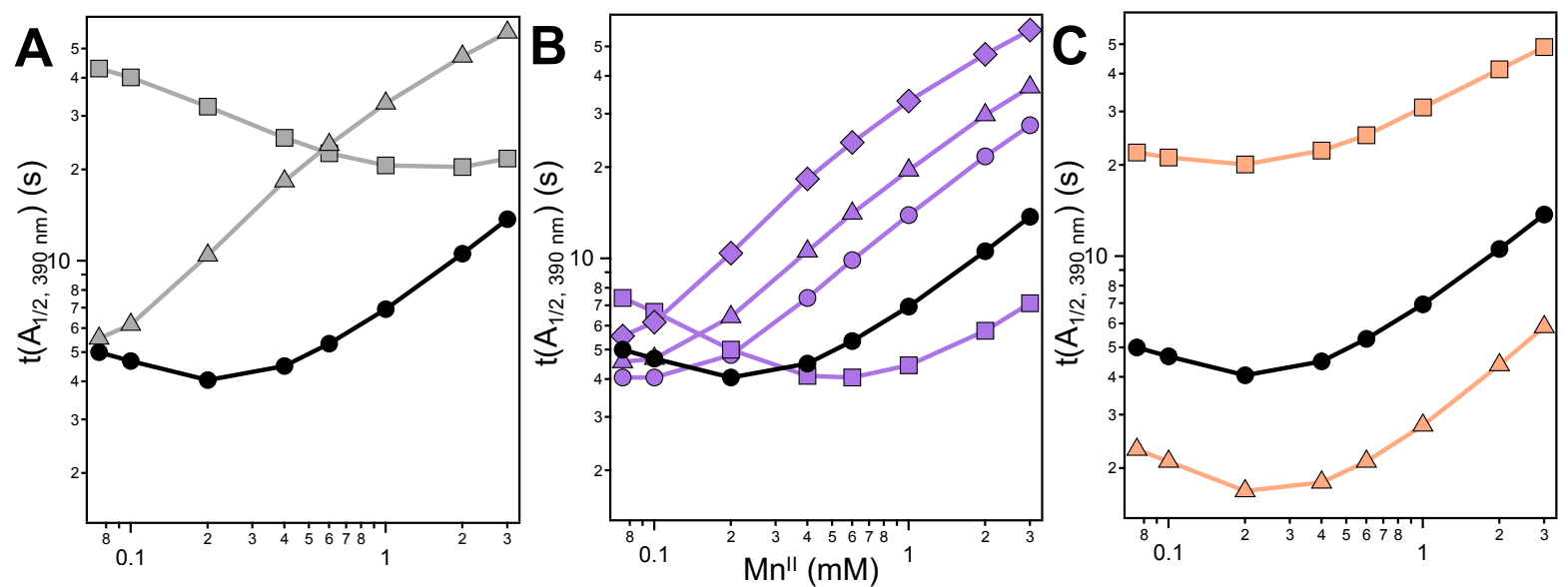

\begin{tabular}{c|c|c|c} 
& $\mathbf{K}_{\mathbf{M n}} \mathbf{M}^{-1}$ & $\mathbf{K}_{\mathbf{F e}} \mathbf{M}^{-1}$ & $\mathbf{K}_{\mathbf{M n}} / \mathbf{K}_{\mathbf{F e}}$ \\
\hline$\triangle$ & $1 \times 10^{5}$ & $1 \times 10^{4}$ & 10 \\
\hline & $\mathbf{1 \times 1 0 ^ { 4 }}$ & $\mathbf{1 \times 1 0 ^ { 3 }}$ & $\mathbf{1 0}$ \\
\hline$\square$ & $1 \times 10^{3}$ & $1 \times 10^{2}$ & 10
\end{tabular}

\begin{tabular}{c|c|c|c} 
& $\mathbf{K}_{\mathbf{M n}} \mathbf{M}^{-1}$ & $\mathbf{K}_{\mathbf{F e}} \mathbf{M}^{-1}$ & $\mathbf{K}_{\mathbf{M n}} / \mathbf{K}_{\mathbf{F e}}$ \\
\hline$\bigcirc$ & $3 \times 10^{4}$ & $1 \times 10^{3}$ & 30 \\
\hline$\triangle$ & $5 \times 10^{4}$ & $1 \times 10^{3}$ & 50 \\
\hline$\triangle$ & $1 \times 10^{5}$ & $1 \times 10^{3}$ & 100 \\
\hline $\mathbf{1 0 1 0 ^ { 4 }}$ & $\mathbf{1 \times 1 0 ^ { 3 }}$ & $\mathbf{1 0}$ \\
\hline$\square$ & $3 \times 10^{3}$ & $1 \times 10^{3}$ & 3
\end{tabular}

\begin{tabular}{c|c|c|c} 
& $\mathbf{K}_{\mathbf{M n}} \mathbf{M}^{-\mathbf{1}}$ & $\mathbf{K}_{\mathbf{F e}} \mathbf{M}^{-\mathbf{1}}$ & $\mathbf{K}_{\mathbf{M n}} / \mathbf{K}_{\mathbf{F e}}$ \\
\hline$\triangle$ & $1 \times 10^{4}$ & $3 \times 10^{3}$ & 3 \\
\hline $\mathbf{1 0}$ & $\mathbf{1 \times 1 0 ^ { 4 }}$ & $\mathbf{1 \times 1 0 ^ { 3 }}$ & $\mathbf{1 0}$ \\
\hline$\square$ & $1 \times 10^{4}$ & $1 \times 10^{2}$ & 100
\end{tabular}

Figure S15. Effect of various metal binding affinities on modeled $t\left(A_{1 / 2}\right)$ values using the competitive/ordered model. Modeled WT data from the competitive/ordered model $\left(\mathrm{K}_{\mathrm{Mn}}=\mathrm{K}^{\prime}{ }_{\mathrm{Mn}}\right.$ $=1 \times 10^{4} \mathrm{M}^{-1}$ and $\mathrm{K}_{\mathrm{Fe}}=1 \times 10^{3} \mathrm{M}^{-1}$ ) shown on each for comparison (black); in all cases, $\mathrm{k}_{\mathrm{Fe} \text {,off }}=$ $\mathrm{k}_{\mathrm{Mn} \text {,off }}^{\prime}=\mathrm{k}_{\mathrm{Mn} \text {,off }}=10 \mathrm{~s}^{-1}$ with the corresponding $\mathrm{k}_{\mathrm{on}}$ adjusted to modulate binding affinities. (A) Ratio of binding affinities remain the same as in the WT model, but each value is adjusted by an order of magnitude up or down. Increasing both of the binding affinities produced a drastic inhibitory effect while decreasing both removes much of the inhibitory effect. (B) $\mathrm{Mn}^{\mathrm{II}} \mathrm{A}$ affinity is modeled at various values. The higher $\mathrm{t}\left(\mathrm{A}_{1 / 2}\right)$ values present at greater $\mathrm{Mn}^{\mathrm{II}}$ concentrations become even more exaggerated as the $\mathrm{K}_{\mathrm{Mn}} / \mathrm{K}_{\mathrm{Fe}}$ ratio increases, i.e., as $\mathrm{K}_{\mathrm{MnA}}$ affinity goes up while $\mathrm{K}_{\mathrm{FeB}}$ is held constant. (C) $\mathrm{Fe}^{\mathrm{II}}{ }_{\mathrm{B}}$ affinity modeled at various values. Changing $\mathrm{K}_{\mathrm{FeB}}$ while holding $\mathrm{K}_{\mathrm{MnA}}$ retains the same inhibition trends seen in $\mathrm{WT}$, with only the absolute $t\left(\mathrm{~A}_{1 / 2}\right)$ values changing. 


\section{$\mathrm{Fe}_{B}$ dependent binding affinity of $\mathrm{Mn}_{\mathrm{A}}$ in the competitive/ordered model}
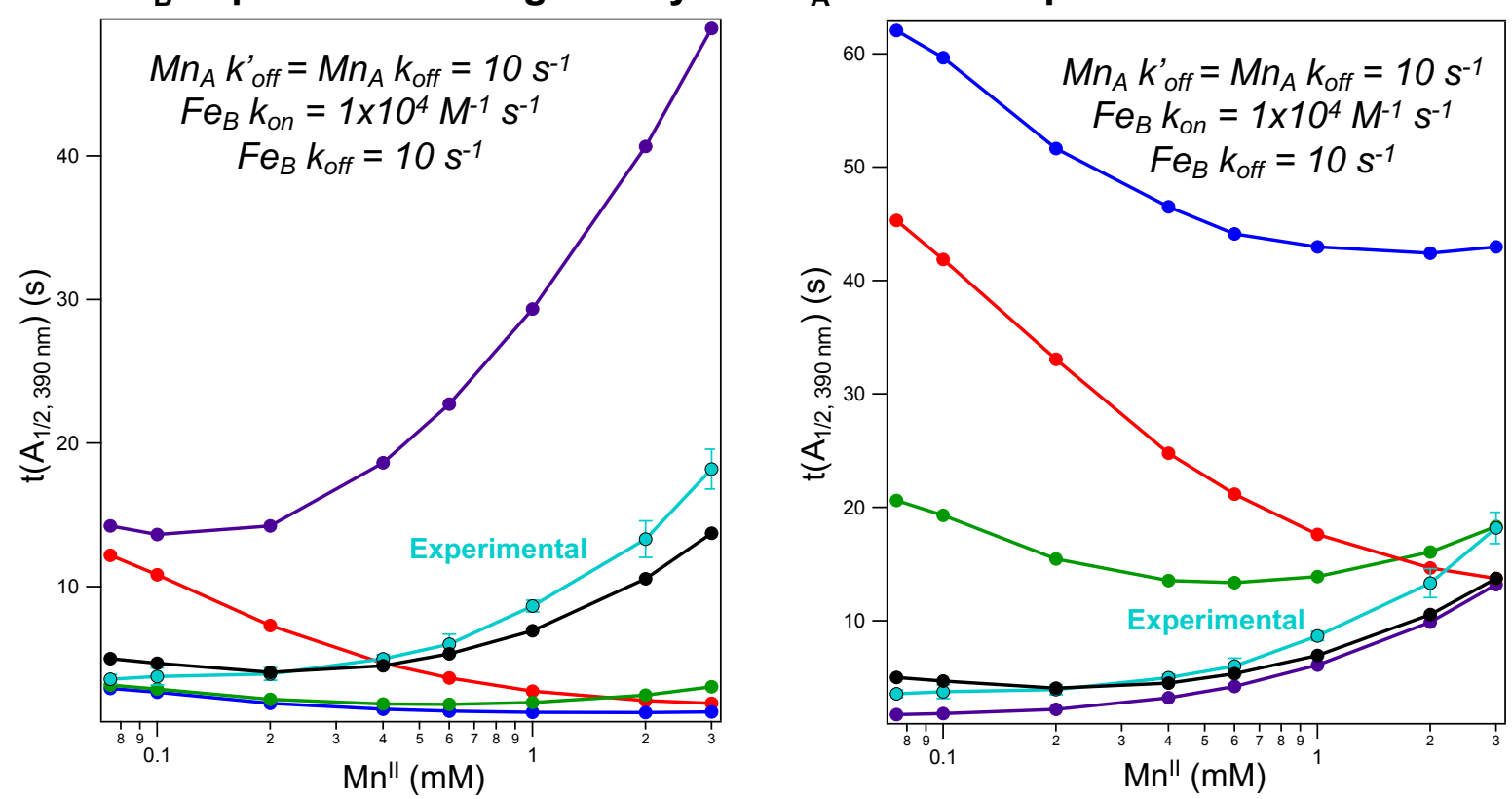

\begin{tabular}{|c|c|c|c|c|c|}
\hline $\begin{array}{c}M n_{A} k_{\text {on }}^{\prime} \\
\left(M^{-1} s^{-1}\right)\end{array}$ & $\begin{array}{l}M_{n_{A}} k_{\text {on }} \\
\left(M^{-1} s^{-1}\right)\end{array}$ & $\begin{array}{l}\mathbf{K}^{\prime}{ }_{\mathrm{MnA}} \\
\left(\mathbf{M}^{-1}\right)\end{array}$ & $\begin{array}{l}K_{\mathrm{MnA}} \\
\left(\mathrm{M}^{-1}\right)\end{array}$ & $\begin{array}{c}\mathbf{K}_{\mathrm{Mn}}^{\prime} \\
\mathrm{K}_{\mathrm{Fe}}\end{array}$ & $\begin{array}{c}\mathrm{K}_{\mathrm{Mn}} / \\
\mathrm{K}_{\mathrm{Fe}}\end{array}$ \\
\hline $1 \times 10^{3}$ & $1 \times 10^{4}$ & $1 \times 10^{2}$ & $1 \times 10^{3}$ & 0.1 & 1 \\
\hline $1 \times 10^{3}$ & $1 \times 10^{5}$ & $1 \times 10^{2}$ & $1 \times 10^{4}$ & 0.1 & 10 \\
\hline $1 \times 10^{4}$ & $1 \times 10^{5}$ & $1 \times 10^{3}$ & $1 \times 10^{4}$ & 1 & 10 \\
\hline $1 \times 10^{6}$ & $1 \times 10^{5}$ & $1 \times 10^{5}$ & $1 \times 10^{4}$ & 100 & 10 \\
\hline $1 \times 10^{5}$ & $1 \times 10^{5}$ & $1 \times 10^{4}$ & $1 \times 10^{4}$ & 10 & 10 \\
\hline
\end{tabular}

\begin{tabular}{|c|c|c|c|c|c|}
\hline $\begin{array}{c}\mathrm{Mn}_{\mathrm{A}} \mathbf{k}_{\text {on }} \\
\left(\mathbf{M}^{-1} \mathbf{s}^{-1}\right)\end{array}$ & $\begin{array}{l}M_{n_{A}} k_{\text {on }} \\
\left(M^{-1} s^{-1}\right) \\
\end{array}$ & $\begin{array}{l}K_{M n A}^{\prime} \\
\left(M^{-1}\right)\end{array}$ & $\begin{array}{l}\mathrm{K}_{\mathrm{MnA}} \\
\left(\mathbf{M}^{-1}\right)\end{array}$ & $\begin{array}{c}\mathbf{K}_{\mathrm{Mn}}^{\prime} \\
\mathbf{K}_{\mathrm{Fe}}\end{array}$ & $\begin{array}{l}\mathrm{K}_{\mathrm{Mn}} l \\
\mathrm{~K}_{\mathrm{Fe}}\end{array}$ \\
\hline $1 \times 10^{4}$ & $1 \times 10^{3}$ & $1 \times 10^{3}$ & $1 \times 10^{2}$ & 1 & 0.1 \\
\hline $1 \times 10^{5}$ & $1 \times 10^{3}$ & $1 \times 10^{4}$ & $1 \times 10^{2}$ & 10 & 0.1 \\
\hline $1 \times 10^{5}$ & $1 \times 10^{4}$ & $1 \times 10^{4}$ & $1 \times 10^{3}$ & 10 & 1 \\
\hline $1 \times 10^{5}$ & $1 \times 10^{6}$ & $1 \times 10^{4}$ & $1 \times 10^{5}$ & 10 & 100 \\
\hline $1 \times 10^{5}$ & $1 \times 10^{5}$ & $1 \times 10^{4}$ & $1 \times 10^{4}$ & 10 & 10 \\
\hline
\end{tabular}

Figure S16. Effect of cooperative binding on modeled $t\left(A_{1 / 2}\right)$ values using the competitive/ordered model. The $\mathrm{Mn}^{\mathrm{II}}$ binding affinity of site $\mathrm{A}$ was adjusted depending on whether site $\mathrm{B}$ was unoccupied $\left(\mathrm{K}^{\prime}{ }_{\mathrm{MnA}}\right)$ or populated with $\mathrm{Fe}^{\mathrm{II}}\left(\mathrm{K}_{\mathrm{MnA}}\right)$. The $\mathrm{k}^{\prime}{ }_{\mathrm{Mn} \text {,off }}$ and $\mathrm{k}_{\mathrm{Mn} \text {,off }}$ rates were both held at 10 $\mathrm{s}^{-1}$ and the $\mathrm{k}_{\mathrm{Mn}, \text { on }}$ or $\mathrm{k}_{\mathrm{Mn}, \mathrm{on}}$ rates adjusted accordingly to give the indicated $\mathrm{K}_{\mathrm{MnA}}$ and $\mathrm{K}_{\mathrm{MnA}}$ values. Experimental data (teal) and $\mathrm{t}\left(\mathrm{A}_{1 / 2}\right)$ values from Figure 4E, i.e., those modeled without considering cooperativity (black), are shown for comparison. To reproduce the experimental trends of increasing $\mathrm{t}\left(\mathrm{A}_{1 / 2}\right)$ values with increasing $\mathrm{Mn}^{\mathrm{II}}$, at least one of the $\mathrm{Mn}^{\mathrm{II}}{ }_{\mathrm{A}}$ binding affinities must be modeled to be greater than $\mathrm{K}_{\mathrm{FeB}}$ with the other modeled to be at least equal to $\mathrm{K}_{\mathrm{FeB}}$ (green, purple). We also note that, although the $t\left(A_{1 / 2}\right)$ values obtained with $\mathrm{K}^{\prime}{ }_{\mathrm{MnA}}>\mathrm{K}_{\mathrm{MnA}}>\mathrm{K}_{\mathrm{FeB}}$ do show inhibition with increasing $\mathrm{Mn}^{\mathrm{II}}$, the modeled $\mathrm{t}\left(\mathrm{A}_{1 / 2}\right)$ values are much greater than those obtained experimentally, while the modeled values obtained when $\mathrm{K}_{\mathrm{MnA}}>\mathrm{K}^{\prime}{ }_{\mathrm{MnA}}>\mathrm{K}_{\mathrm{FeB}}$ are much closer to those of the experimental inhibition curve. 


\section{$\mathrm{Fe}_{\mathrm{B}}$ dependent binding affinity of $\mathrm{Mn}_{\mathrm{A}}$ in the competitive/ordered model with faster metal association/dissociation rates}
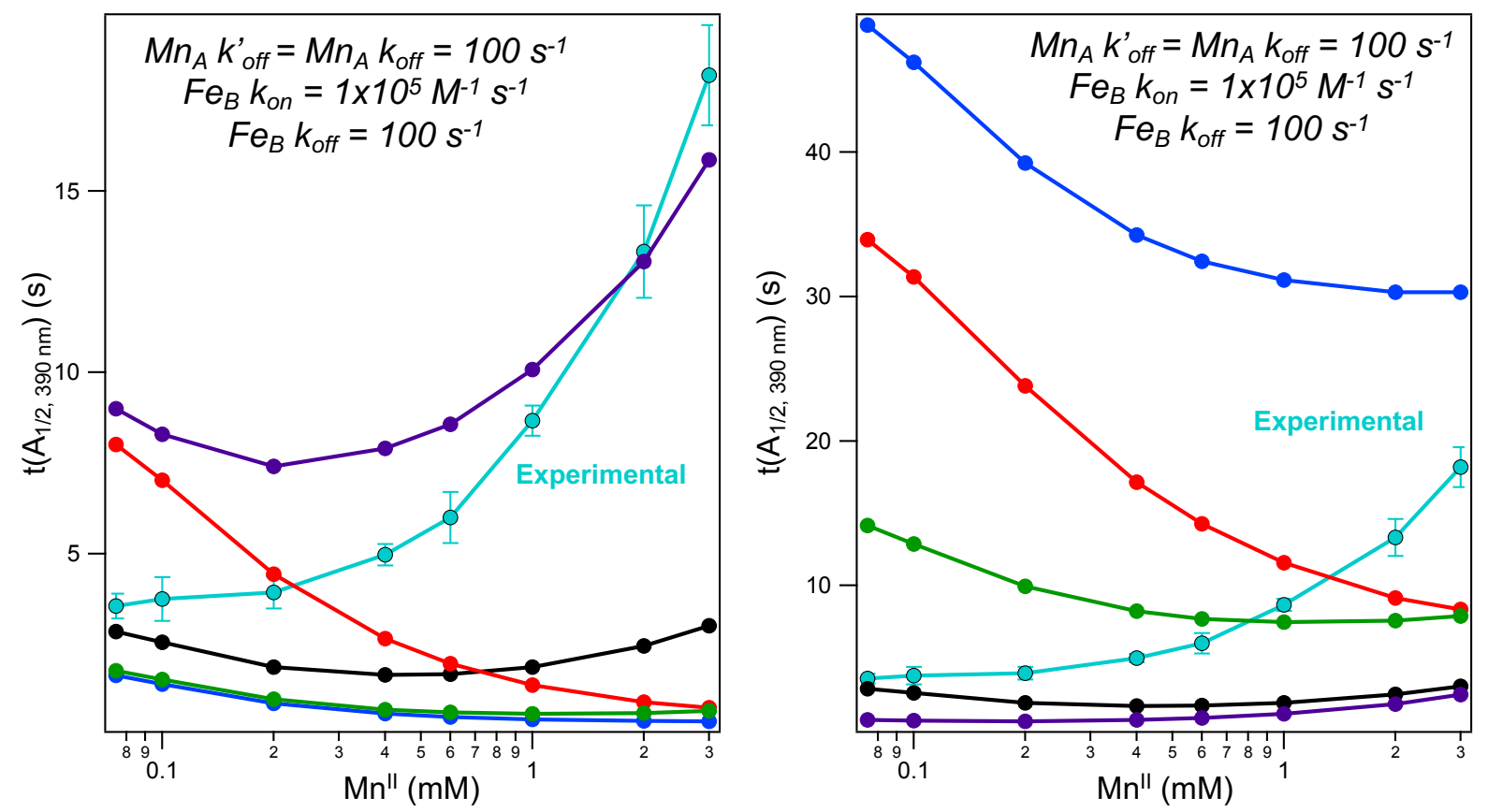

\begin{tabular}{|c|c|c|c|c|c|}
\hline $\begin{array}{c}\mathbf{M n}_{\mathbf{A}} \mathbf{K}_{\text {on }}^{\prime} \\
\mathbf{( M}^{-1} \mathbf{s}^{-1} \mathbf{)}\end{array}$ & $\begin{array}{c}\mathbf{M n}_{\mathbf{A}} \mathbf{K}_{\mathbf{o n}} \\
\mathbf{( M}^{-1} \mathbf{s}^{-1} \mathbf{)}\end{array}$ & $\begin{array}{c}\mathbf{K}_{\mathbf{M n A}}^{\prime} \\
\mathbf{( M}^{-1} \mathbf{)}\end{array}$ & $\begin{array}{c}\mathbf{K}_{\mathbf{M n A}} \\
\mathbf{( M}^{-1} \mathbf{)}\end{array}$ & $\begin{array}{c}\mathbf{K}_{\mathbf{M n}}^{\prime} / \\
\mathbf{K}_{\mathbf{F e}}\end{array}$ & $\begin{array}{c}\mathbf{K}_{\mathbf{M n}} / \\
\mathbf{K}_{\mathbf{F e}}\end{array}$ \\
\hline $1 \times 10^{4}$ & $1 \times 10^{5}$ & $1 \times 10^{2}$ & $1 \times 10^{3}$ & 0.1 & 1 \\
\hline $1 \times 10^{4}$ & $1 \times 10^{6}$ & $1 \times 10^{2}$ & $1 \times 10^{4}$ & 0.1 & 10 \\
\hline $1 \times 10^{5}$ & $1 \times 10^{6}$ & $1 \times 10^{3}$ & $1 \times 10^{4}$ & 1 & 10 \\
\hline $1 \times 10^{7}$ & $1 \times 10^{6}$ & $1 \times 10^{5}$ & $1 \times 10^{4}$ & 100 & 10 \\
\hline $1 \times 10^{6}$ & $1 \times 10^{6}$ & $1 \times 10^{4}$ & $1 \times 10^{4}$ & 10 & 10 \\
\hline
\end{tabular}

\begin{tabular}{|c|c|c|c|c|c|}
\hline $\begin{array}{c}\mathbf{M n}_{\mathbf{A}} \mathbf{k}_{\text {on }}^{\prime} \\
\left.\mathbf{( M}^{-1} \mathbf{s}^{-1}\right)\end{array}$ & $\begin{array}{c}\mathbf{M n}_{\mathbf{A}} \mathbf{k}_{\text {on }} \\
\left.\mathbf{( M}^{-1} \mathbf{s}^{-1}\right)\end{array}$ & $\begin{array}{c}\mathbf{K}_{\mathbf{M n A}}^{\prime} \\
\left.\mathbf{( M}^{-1}\right)\end{array}$ & $\begin{array}{c}\mathbf{K}_{\mathbf{M n A}} \\
\mathbf{( M}^{-1} \mathbf{)}\end{array}$ & $\begin{array}{c}\mathbf{K}_{\mathbf{M n}}^{\prime} / \\
\mathbf{K}_{\mathbf{F e}}\end{array}$ & $\begin{array}{c}\mathbf{K}_{\mathbf{M n}} / \\
\mathbf{K}_{\mathbf{F e}}\end{array}$ \\
\hline $1 \times 10^{5}$ & $1 \times 10^{4}$ & $1 \times 10^{3}$ & $1 \times 10^{2}$ & 1 & 0.1 \\
\hline $1 \times 10^{6}$ & $1 \times 10^{4}$ & $1 \times 10^{4}$ & $1 \times 10^{2}$ & 10 & 0.1 \\
\hline $1 \times 10^{6}$ & $1 \times 10^{5}$ & $1 \times 10^{4}$ & $1 \times 10^{3}$ & 10 & 1 \\
\hline $1 \times 10^{6}$ & $1 \times 10^{7}$ & $1 \times 10^{4}$ & $1 \times 10^{5}$ & 10 & 100 \\
\hline $1 \times 10^{6}$ & $1 \times 10^{6}$ & $1 \times 10^{4}$ & $1 \times 10^{4}$ & 10 & 10 \\
\hline
\end{tabular}

Figure S17. Effect of increasing metal association/dissociation $\left(\mathrm{k}_{\mathrm{on}} / \mathrm{k}_{\mathrm{off}}\right)$ rates on modeled $\mathrm{t}\left(\mathrm{A}_{1 / 2}\right)$ values using the models shown in Figure S16, i.e. the competitive/ordered model (black traces) or the competitive/ordered model with cooperativity (colored traces). For all models shown in Figure $\mathrm{S} 16$, the $\mathrm{k}_{\text {on }} / \mathrm{k}_{\text {off }}$ rates were increased by an order of magnitude. Experimental data (teal) are shown for comparison. With $\mathrm{k}^{\prime}{ }_{\mathrm{Mn}, \text { off }}=\mathrm{k}_{\mathrm{Mn}, \text { off }}=\mathrm{k}_{\mathrm{Fe}, \text { off }}=100 \mathrm{~s}^{-1}$, none of the attempted models fully reproduce the experimental data. 


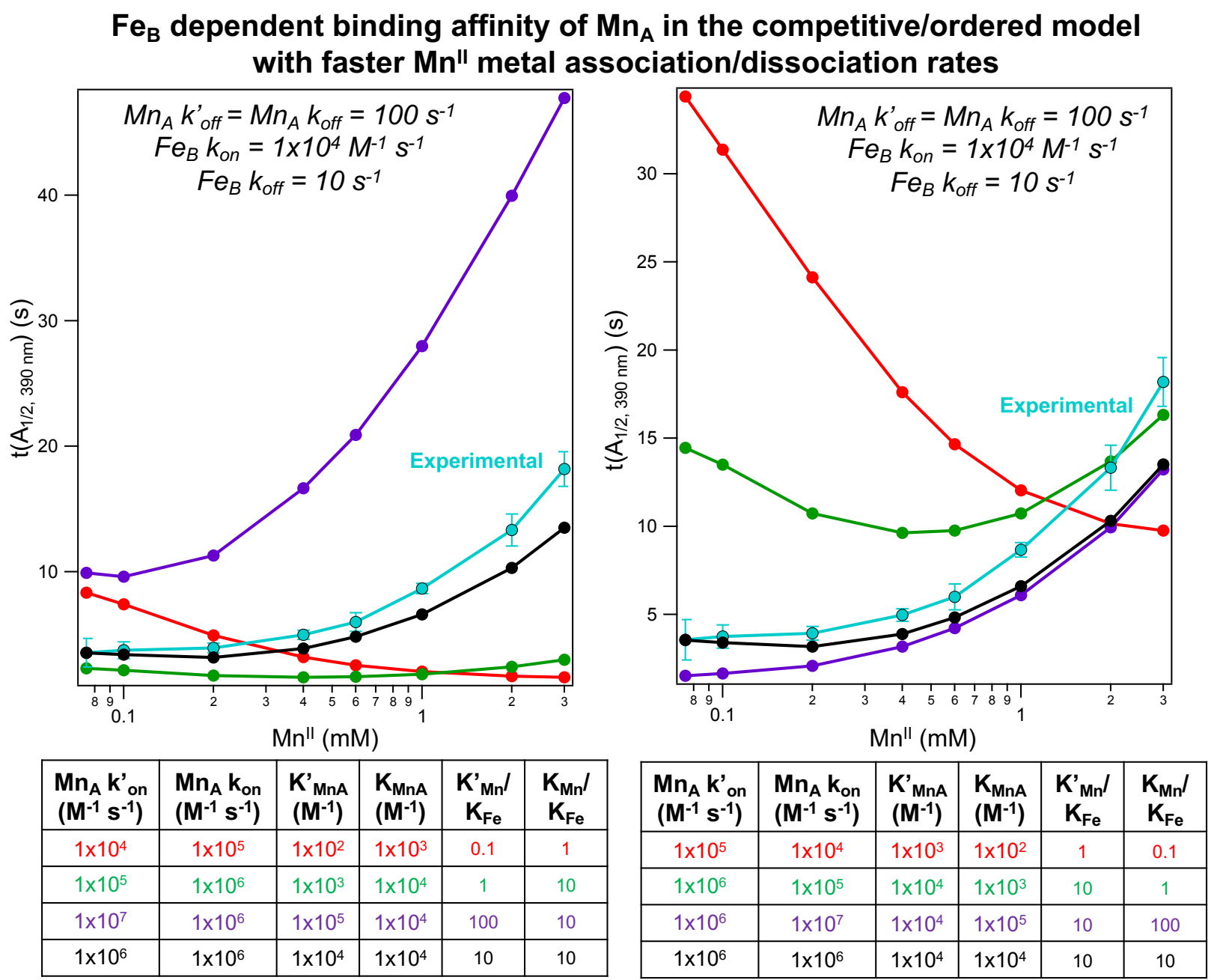

Figure S18. Effect of increasing only $\mathrm{Mn}_{\mathrm{A}}^{\mathrm{II}}$ association/dissociation $\left(\mathrm{k}_{\mathrm{o}} / \mathrm{k}_{\text {off }}\right)$ rates on modeled $\mathrm{t}\left(\mathrm{A}_{1 / 2}\right)$ values using the models shown in Figure S16, i.e. the competitive/ordered model (black traces) or the competitive/ordered model with cooperativity (colored traces). The $\mathrm{k}^{\mathrm{Mn} \text {,off }}$ and $\mathrm{k}_{\mathrm{Mn}, \mathrm{off}}$ rates were both held at $100 \mathrm{~s}^{-1}$ in all cases, and the $\mathrm{k}^{\prime} \mathrm{Mn}$,on and $\mathrm{k}_{\mathrm{Mn}, \mathrm{on}}$ rates were adjusted to give the indicated $\mathrm{K}^{\prime}{ }_{\mathrm{MnA}}$ and $\mathrm{K}_{\mathrm{MnA}}$ values. Experimental data (teal) is shown for comparison. When at least one of the $\mathrm{Mn}^{\mathrm{II}}$ binding affinities is weaker than $\mathrm{K}_{\mathrm{FeB}}($ red), no inhibition is observed. When at least one of the $\mathrm{Mn}^{\text {II }}$ binding affinities is greater than $\mathrm{K}_{\mathrm{FeB}}$ (green), some degree of inhibition is observed, but with different trends than the experimental. Only when both $\mathrm{Mn}^{\mathrm{II}}$ binding affinities are greater than $\mathrm{K}_{\mathrm{FeB}}$ are inhibition curves similar to the experimental curves observed (purple, black), although if $\mathrm{K}^{\prime}{ }_{\mathrm{MnA}}>\mathrm{K}_{\mathrm{MnA}}>\mathrm{K}_{\mathrm{FeB}}$ (purple, left), the modeled inhibition is significantly greater than that observed experimentally. 

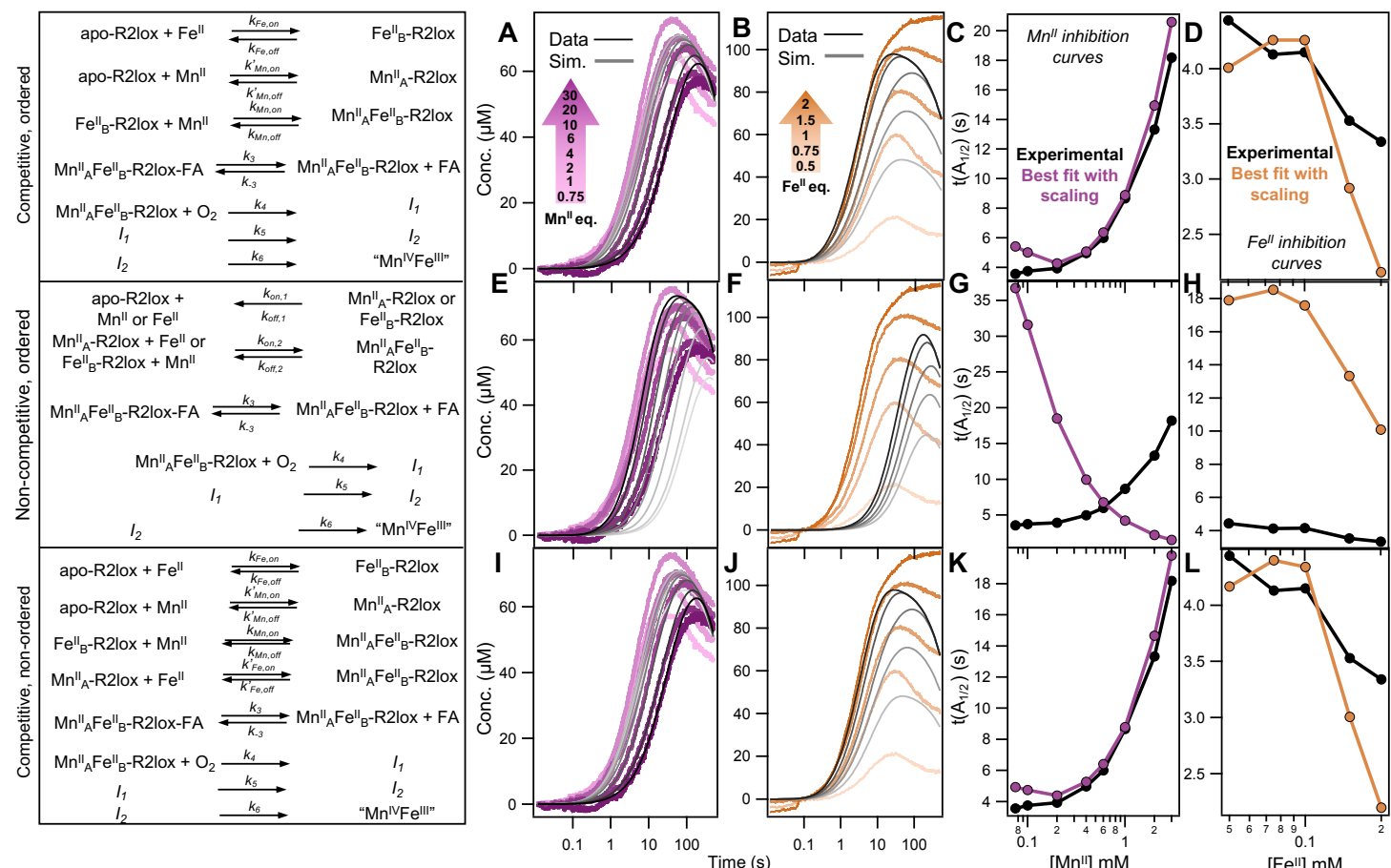

\begin{tabular}{|c|c|c|c|c|c|c|c|c|c|c|c|c|c|c|c|c|c|c|}
\hline & & \multicolumn{2}{|c|}{$[\mathrm{Mn} "] \mathrm{mM}$} & \multicolumn{3}{|c|}{$\left[\mathrm{Fe}^{\prime \prime}\right] \mathrm{mM}$} & \multirow[b]{2}{*}{$\begin{array}{l}\mathbf{k}_{6} \\
\mathbf{s}^{-1}\end{array}$} \\
\hline & $\begin{array}{l}\mathbf{k}_{\text {Fe,on }} \\
\mathbf{M}^{-1} \mathbf{s}^{-1}\end{array}$ & $\mathbf{k}_{\mathrm{Fe}_{\mathrm{e}, \text { off }}}$ & $\begin{array}{l}\mathbf{K}_{\text {fe }} \\
\mathbf{M}^{-1}\end{array}$ & $\begin{array}{l}\mathbf{k}_{M, n, o n}^{\prime} \\
\mathbf{M}^{-1} \mathbf{s}^{-1}\end{array}$ & $\mathbf{k}_{\mathbf{S}_{\mathbf{S}, 1}^{\prime}-\text { off }}^{\prime}$ & $\mathbf{K}_{\mathbf{M}-1}^{\prime}$ & $\begin{array}{l}\mathbf{k}_{\mathrm{Mn}, 00} \\
\mathbf{M}^{-1}, \mathbf{s}^{-1}\end{array}$ & $\begin{array}{c}k_{\text {Mn,off }} \\
\mathbf{s}^{-1}\end{array}$ & $\begin{array}{l}\mathbf{K}_{\mathbf{M n}} \\
\mathbf{M}^{-1}\end{array}$ & $\begin{array}{l}\mathbf{k}_{\text {Fee, on }} \\
\mathbf{M}^{-1} \mathbf{S}^{-1}\end{array}$ & $\begin{array}{c}\mathbf{k}_{\text {Fe,offf }}^{\prime} \\
\mathbf{s}^{-1}\end{array}$ & $\begin{array}{l}\mathbf{K}_{\mathrm{Fe}}^{\prime} \\
\mathbf{M}^{-1}\end{array}$ & $\begin{array}{l}\mathbf{k}_{3} \\
\mathbf{s}^{-1}\end{array}$ & $\begin{array}{l}\mathbf{k}_{-3} \\
\mathbf{s}^{-1}\end{array}$ & $K_{\text {FA }}$ & $\begin{array}{l}\mathbf{k}_{4} \\
\mathbf{s}^{-1}\end{array}$ & $\begin{array}{l}\mathbf{k}_{5} \\
\mathbf{s}^{-1}\end{array}$ & \\
\hline $\mathrm{c} / \mathrm{O}$ & $\begin{array}{c}9720 \\
\pm \\
157\end{array}$ & $\begin{array}{c}21.7 \\
\pm .3 \\
14.3\end{array}$ & $\begin{array}{c}448 \\
\pm \\
298\end{array}$ & $\begin{array}{c}1.54 \times 10^{6} \\
\pm 1100\end{array}$ & $\begin{array}{c}163 \\
17.6 \\
17.6\end{array}$ & $\begin{array}{c}9460 \\
\pm \\
1060\end{array}$ & $\begin{array}{c}1.41 \times 10^{5} \\
\pm \pm 14 \times 10^{5} \\
1.1\end{array}$ & $\begin{array}{c}0.209 \\
\pm .3 \\
25.3\end{array}$ & $\begin{array}{c}6.75 \times 10^{6} \\
1 \pm 16 \times 10^{6}\end{array}$ & - & - & & $\begin{array}{c}25.5 \\
9.8 \\
93.8\end{array}$ & $\begin{array}{c}1.116 \\
\pm \\
899\end{array}$ & $\begin{array}{c}22 \\
\pm \\
17200\end{array}$ & $\begin{array}{c}1.97 \times 10^{6} \\
\pm \\
4.81 \times 10^{7}\end{array}$ & $\begin{array}{c}13.3 \\
\pm \\
14.1\end{array}$ & $\begin{array}{c}79600 \\
\pm \\
\text { ND }\end{array}$ \\
\hline $\mathrm{C} / \mathrm{NO}$ & $\begin{array}{c}4480 \\
\pm \\
1120 \\
\end{array}$ & $\begin{array}{c}86.5 \\
\pm \\
340\end{array}$ & $\begin{array}{c}52 \\
\pm \\
204 \\
\end{array}$ & $\begin{array}{r}1.69 \times 10^{5} \\
2.00 \times 10^{5} \\
2\end{array}$ & $\begin{array}{c}41.1 \\
\pm \\
49.4 \\
\end{array}$ & $\begin{array}{c}4130 \\
\pm \pm \\
6960 \\
\end{array}$ & $\begin{array}{l}1.56 \times 10^{6} \\
3.12 \times 10^{7} \\
\end{array}$ & $\begin{array}{c}543 \\
7.91 \times 10^{4} \\
\end{array}$ & $\begin{array}{c}2870 \\
4.23 \times 10^{5} \\
\end{array}$ & $\begin{array}{c}0.934 \\
7.11 \\
\end{array}$ & $\begin{array}{c}0 . \\
026 \\
\pm \mathrm{ND} \\
\end{array}$ & $\begin{array}{l}36 \\
\pm \\
\text { ND } \\
\end{array}$ & $\begin{array}{c}2400 \\
\pm \\
1.24 \times 10^{5} \\
\end{array}$ & $\begin{array}{c}8.23 \\
\pm \\
749 \\
\end{array}$ & $\begin{array}{c}292 \\
\pm \pm 000 \\
30600 \\
\end{array}$ & $\begin{array}{c}1.22 \times 10^{6} \\
\pm \\
1.38 \times 10^{7}\end{array}$ & $\begin{array}{c}8.02 \\
\pm \\
1.06 \\
\end{array}$ & $\begin{array}{c}79800 \\
\pm \\
\text { ND } \\
\end{array}$ \\
\hline & $k_{o n, 1}$ & $k_{\text {off, } 1}$ & $K_{M, 1}$ & $k_{\mathrm{on}, 2}$ & $k_{\text {off }, 2}$ & $\mathbf{K}_{M, 2}$ & - & - & - & - & - & - & $k_{3}$ & $k_{-3}$ & $\mathrm{~K}_{\mathrm{FA}}$ & $k_{4}$ & $k_{5}$ & $k_{6}$ \\
\hline $\mathrm{NC} / \mathrm{O}$ & $\begin{array}{c}4.03 \times 10^{5} \\
\pm \pm 10^{6} \\
1.95 \times 10^{6}\end{array}$ & $\begin{array}{c}16500 \\
\pm 000\end{array}$ & $\begin{array}{c}24.4 \\
\pm \\
168\end{array}$ & $\begin{array}{c}84300 \\
\pm \\
24600\end{array}$ & $\begin{array}{c}22.5 \\
\pm \\
39200\end{array}$ & $\begin{array}{c}3750 \\
\pm \\
6.53 \times 10^{6}\end{array}$ & - & . & - & - & - & . & $\begin{array}{l}1.33 \times 10^{5} \\
2.33 \times 10^{8}\end{array}$ & $\begin{array}{c}33.6 \\
\pm \\
1.13 \times 10^{5}\end{array}$ & $\begin{array}{c}3960 \\
1.50 \times 10^{7}\end{array}$ & $\begin{array}{c}1.97 \times 10^{6} \\
\stackrel{ \pm}{ \pm} \times 10^{7}\end{array}$ & $\begin{array}{c}13.3 \\
\pm \\
14.1\end{array}$ & $\begin{array}{c}79600 \\
\pm \\
\text { ND }\end{array}$ \\
\hline
\end{tabular}

Figure S19. $I_{2}$ formation during SF metal competition experiments in WT R2lox was modeled using KinTek (grey traces) and fit to the $\mathrm{Mn}^{\mathrm{II}}$ (purple) and $\mathrm{Fe}^{\mathrm{II}}$ (orange) experimental data through the use of a 20-fold scaling factor. Multiple models were considered (left column), with the exception of the competitive, non-ordered, and site non-selective model, based on the lack of experimental evidence for population of the $\mathrm{B}$ site by $\mathrm{Mn}^{\mathrm{II}}$. The fundamental rate constants for each model and their standard errors were obtained by allowing the model to vary all parameters in order to globally optimize the fit to all of the scaled $\mathrm{Mn}^{\mathrm{II}}$ and $\mathrm{Fe}^{\mathrm{II}}$ competition data at once. Corresponding $\mathrm{t}\left(\mathrm{A}_{1 / 2}\right)$ values were obtained from the modeled data for comparison with the experimental and metal inhibition curves generated. 

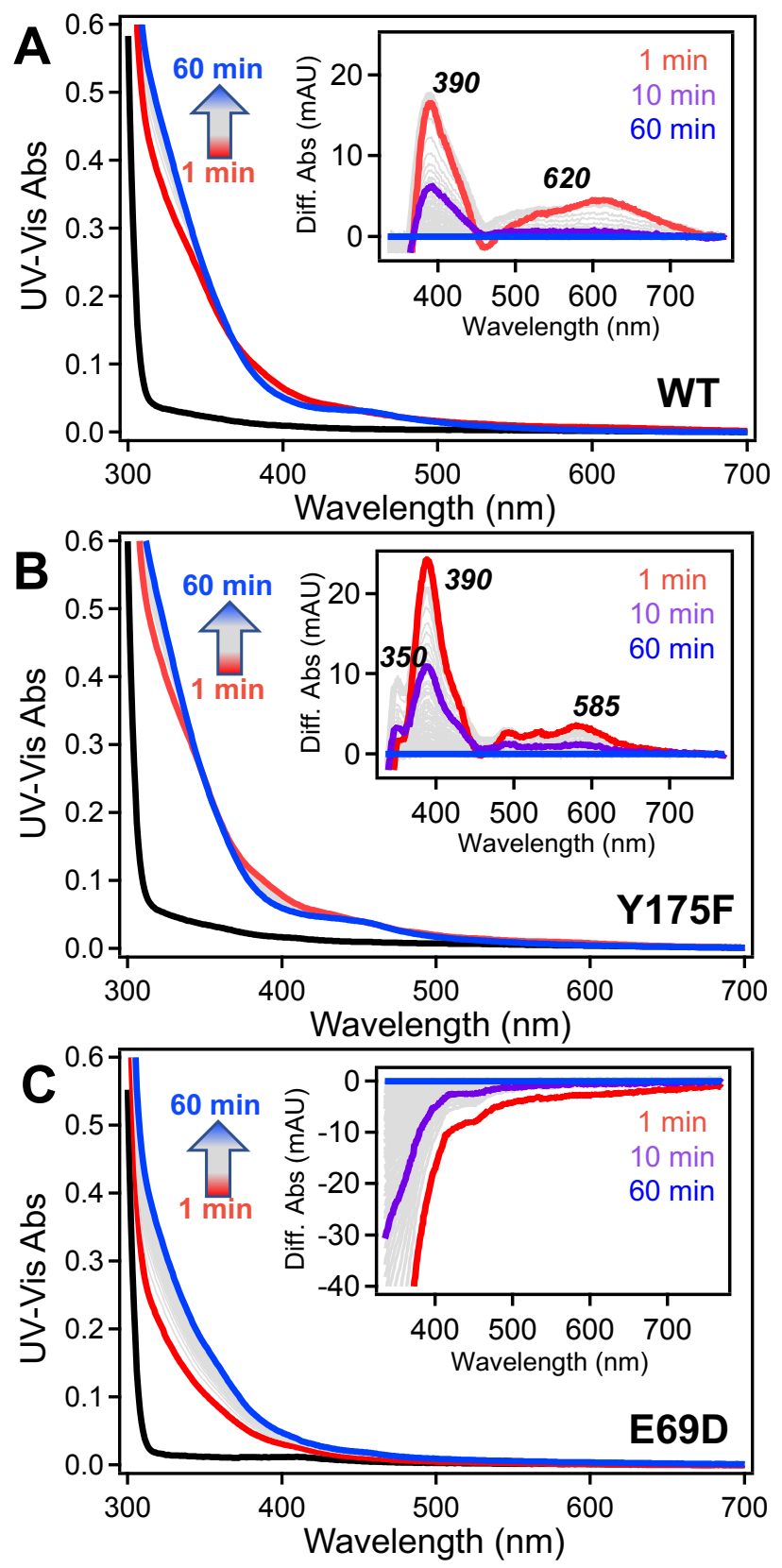

Figure S20. Time-resolved UV-vis spectra of $100 \mu \mathrm{M}$ (A) apo WT (B) apo Y175F and (C) apo E69D R2lox (black) following addition of $75 \mu \mathrm{M}$ each $\mathrm{Mn}^{\mathrm{II}}$ and $\mathrm{Fe}^{\mathrm{II}}$ under aerobic conditions, from approx. $1 \mathrm{~min}$ (red) to $60 \mathrm{~min}$ (blue). (Insets) Difference absorption spectra $\left(\mathrm{t}_{\mathrm{x}}-\mathrm{t}_{\mathrm{final}}\right)$ of each R2lox variant with select timepoints highlighted. In Y175F, the WT-like absorption feature at 390 $\mathrm{nm}$ is still observed, with a more pronounced shoulder at $350 \mathrm{~nm}$. 


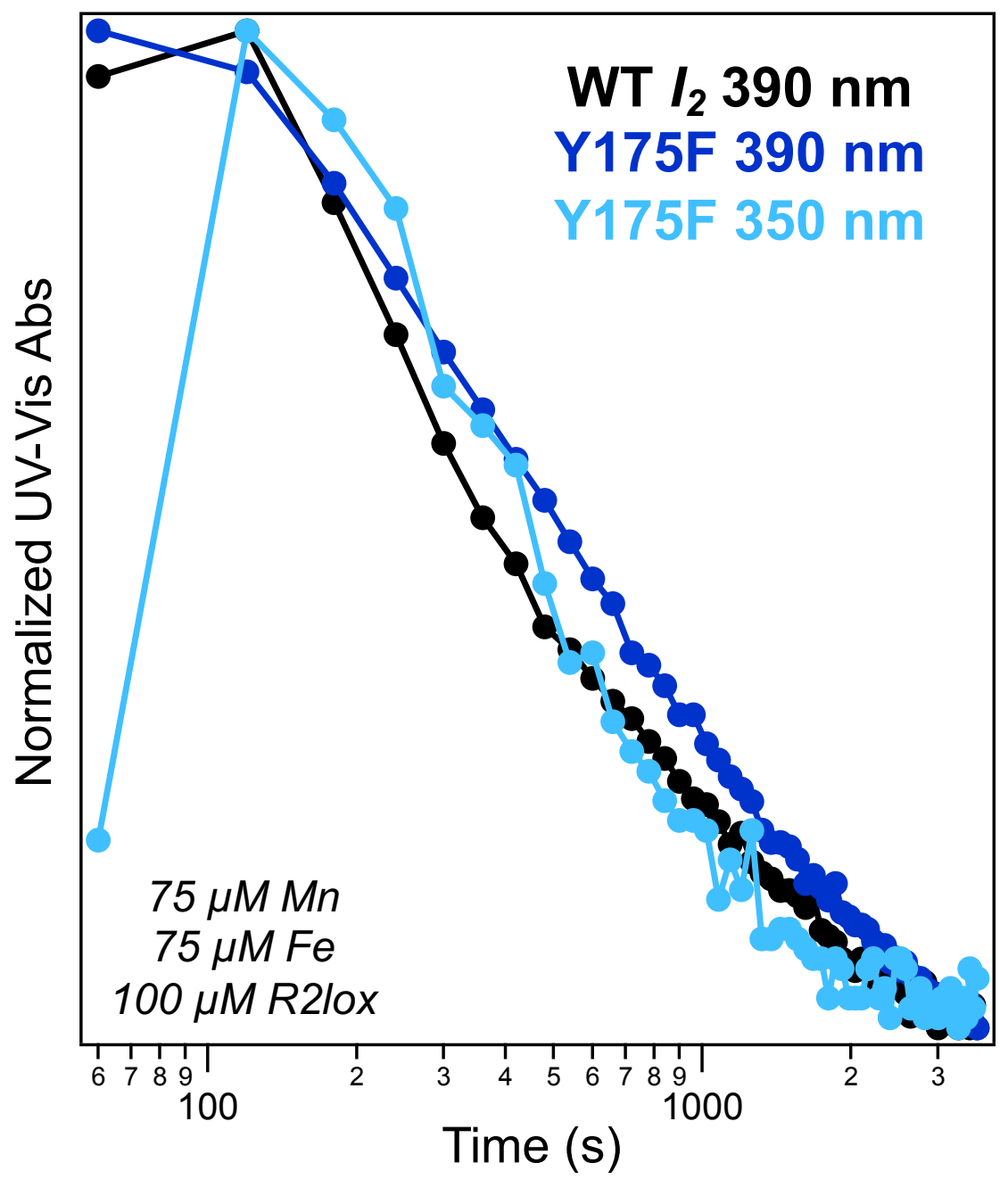

Figure S21. Comparison of UV-vis intermediate decay kinetics in WT and Y175F at the indicated wavelengths. Kinetic traces taken from metallations performed using $100 \mu \mathrm{M}$ protein with $75 \mu \mathrm{M}$ each of $\mathrm{Mn}^{\mathrm{II}}$ and $\mathrm{Fe}^{\mathrm{II}}$ (as in Figure S20). Data are shown normalized for intensity to directly compare kinetics of decay. Time shown in logarithmic scale. Decay kinetics of both $350 \mathrm{~nm}$ and $390 \mathrm{~nm}$ in Y175F track with those of WT at $390 \mathrm{~nm}$, albeit at a slightly slower $(\sim 20 \%)$ rate in Y175F than WT. 


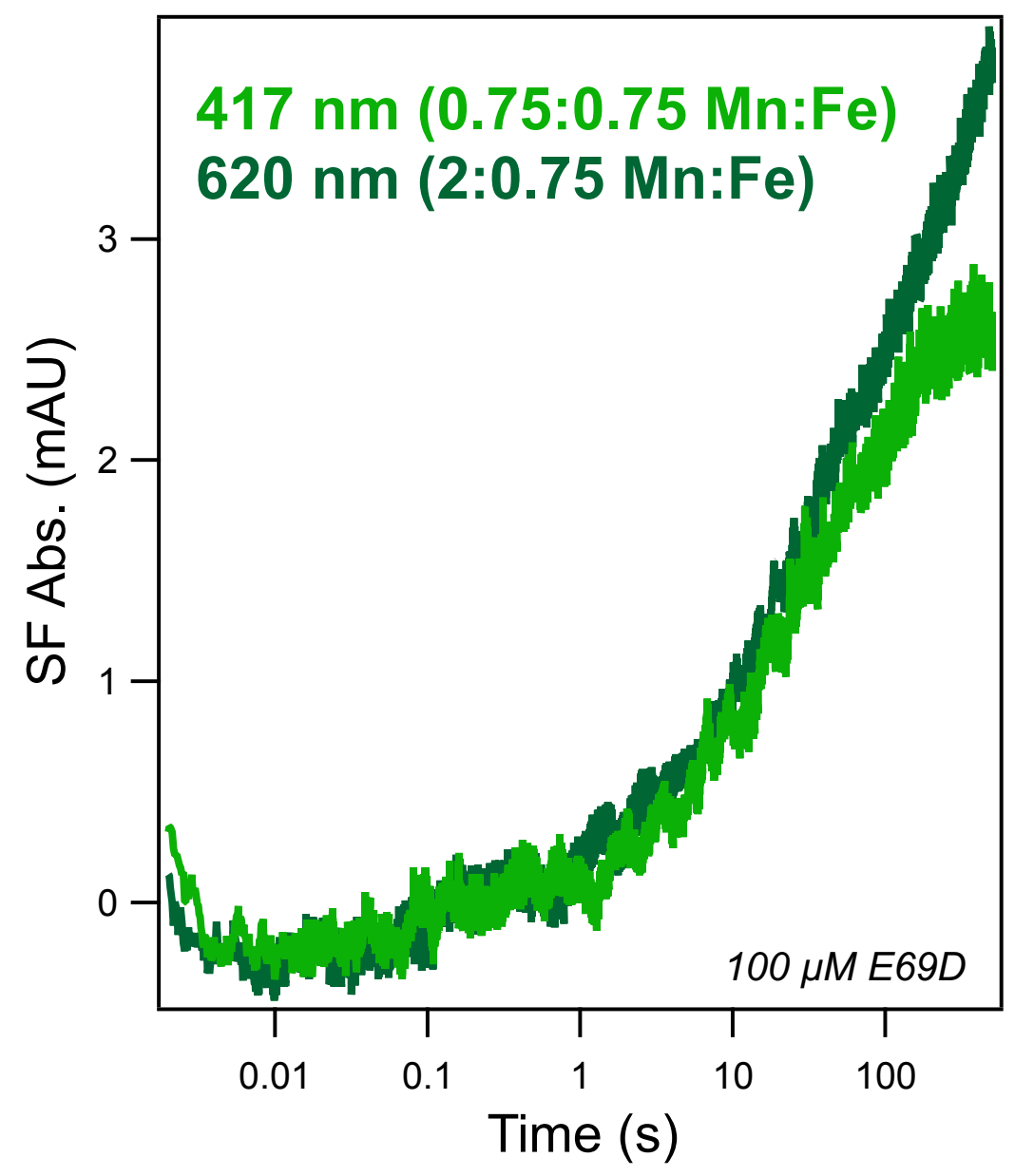

Figure S22. SF traces monitoring E69D Mn/Fe cofactor formation at indicated wavelengths for intermediate formation, analogous to those seen in WT and Y175F. 


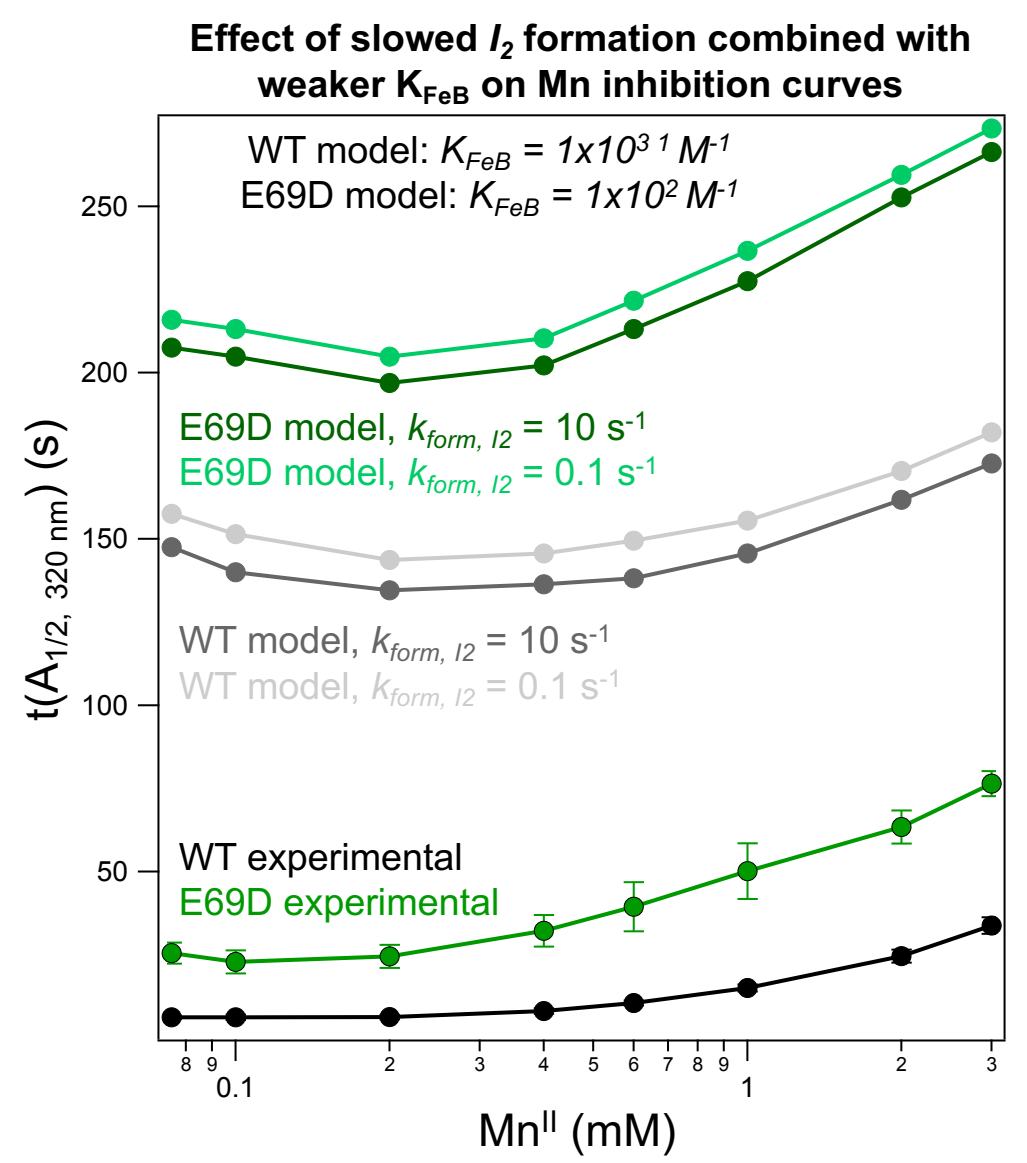

Figure S23. Effect of a slower $I_{2}$ formation rate on modeled $\mathrm{t}\left(\mathrm{A}_{1 / 2}\right)$ values using the WT $\left(K_{F e B}=\right.$ $\left.10^{3} \mathrm{M}^{-1}\right)$ or E69D $\left(K_{F e B}=10^{2} M^{-1}\right)$ competitive/ordered models. Both experimental inhibition curves obtained from $320 \mathrm{~nm}$ (resting state) data (black, green) and $\mathrm{t}\left(\mathrm{A}_{1 / 2}\right)$ values obtained from the modeled resting state species without slowed $I_{2}$ formation $\left(\mathrm{k}_{5}=10 \mathrm{~s}^{-1}\right)$ (dark grey, dark green) are shown for comparison. For $\mathrm{Fe}^{\mathrm{II}}$ binding to site $\mathrm{B}, \mathrm{k}_{\mathrm{Fe} \text {,off }}$ values were held at $10 \mathrm{~s}^{-1}$ and $\mathrm{k}_{\mathrm{Fe} \text {,on }}$ modified. A slowed $I_{2}$ decay rate of $\mathrm{k}_{5}=0.1 \mathrm{~s}^{-1}$ (light grey, light green) results in $\mathrm{t}\left(\mathrm{A}_{1 / 2}\right)$ values that are slightly longer but does not significantly alter the shape of the inhibition curves. Therefore, a slowed rate of formation for $I_{2}$ is not sufficient to reproduce the E69D inhibition curve, which has longer $t\left(\mathrm{~A}_{1 / 2}\right)$ values but also exhibits a slightly greater inhibitory effect with increasing $\mathrm{Mn}^{\mathrm{II}}$ relative to WT. Only when a weaker $\mathrm{K}_{\mathrm{FeB}}$ is invoked, i.e., in the E69D model, are both features of the E69D experimental data reproduced. 


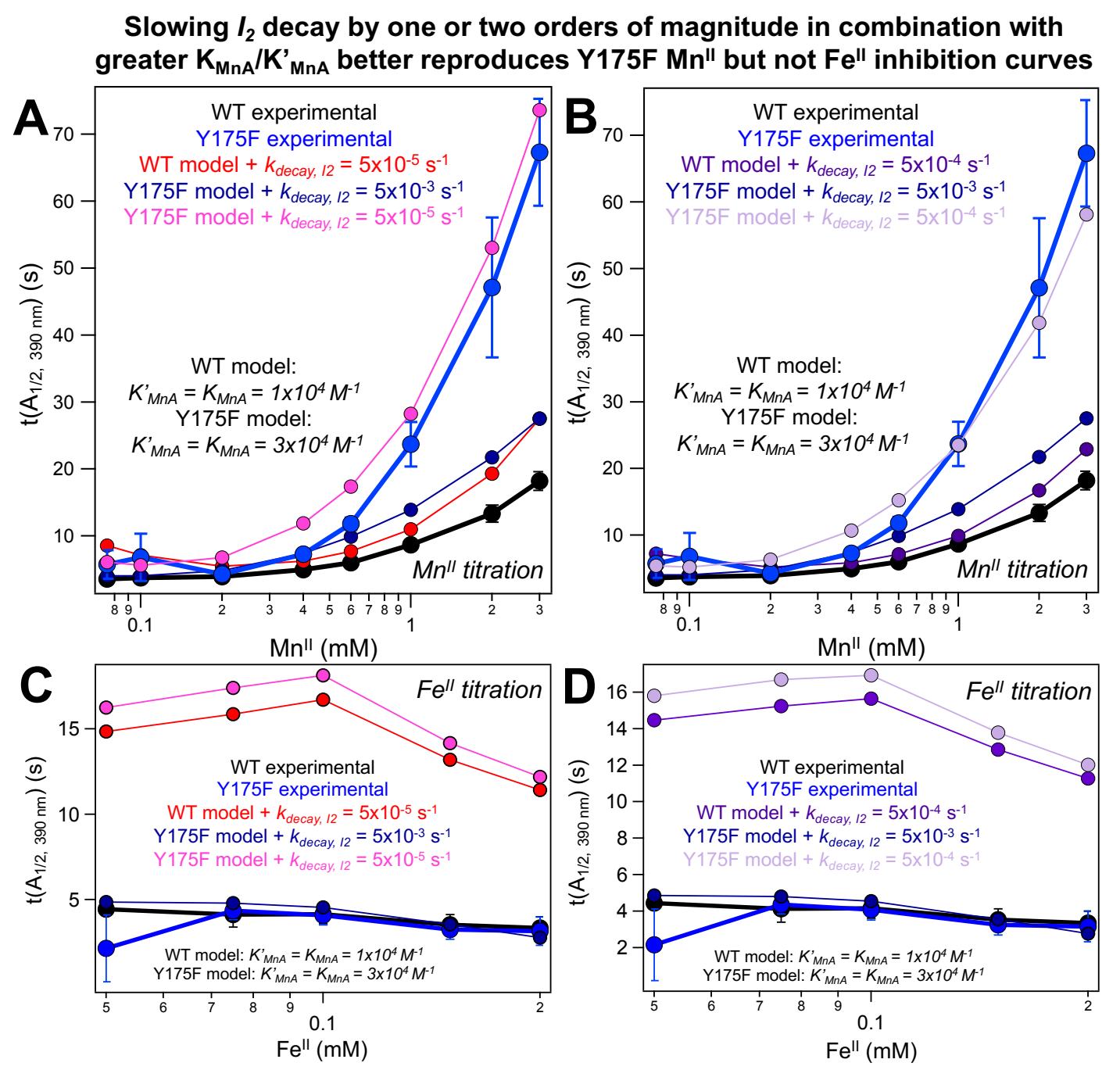

Figure S24. Effects of a slower $I_{2}$ decay rate on modeled $\mathrm{t}\left(\mathrm{A}_{1 / 2}\right)$ values using the WT $\left(K_{M n A}=\right.$ $\left.K^{\prime}{ }_{M n A}=10^{4} M^{-1}\right)$ or Y $175 \mathrm{~F}\left(K_{M n A}=K^{\prime}{ }_{M n A}=3 \times 10^{4} M^{-1}\right)$ competitive/ordered models to generate $\mathrm{Mn}^{\mathrm{II}}$ (top) and $\mathrm{Fe}^{\mathrm{II}}$ (bottom) inhibition curves. Both experimental data (black, blue) and modeled $\mathrm{t}\left(\mathrm{A}_{1 / 2}\right)$ values obtained from the Y175F model without slowed $I_{2}$ decay $\left(\mathrm{k}_{6}=5 \mathrm{x} 10^{-3} \mathrm{~s}^{-1}\right)($ dark blue) are shown for comparison. For $\mathrm{Mn}^{\mathrm{II}}$ binding to site $\mathrm{A}, \mathrm{k}_{\text {off }}$ values were held at $10 \mathrm{~s}^{-1}$ and the corresponding $\mathrm{k}_{\mathrm{on}}$ modified. A slowed $I_{2}$ decay rate of either $\mathrm{k}_{6}=5 \times 10^{-5} \mathrm{~s}^{-1}$ (left) or $\mathrm{k}_{6}=5 \times 10^{-4}$ $\mathrm{s}^{-1}$ (right) was applied to both the WT (red, purple) and Y175F (magenta, light purple) models. While using a slower rate of $I_{2}$ decay in the Y175F model better reproduces the experimental $\mathrm{Y} 175 \mathrm{~F} \mathrm{Mn}{ }^{\mathrm{II}}$ inhibition curve, it has the opposite effect regarding the $\mathrm{Fe}^{\mathrm{II}}$ inhibition curves. This likely indicates the Y175F mutation also perturbs one or more rate constants within the assembly mechanism in addition to modulating the binding affinity of site A. Although these implied intermediate species are invoked within the kinetic model, many do not yet have clearly identified and/or isolated spectroscopic handles to monitor, making it intractable to thoroughly assess how these individual changes might affect the current model. While the in-depth investigation of the Y175F assembly pathway is currently being pursued in our lab, a rigorous examination of the perturbation to the assembly mechanism is beyond the scope of this work. As such, we have attempted to assess only the effects on metal binding affinity. 
Effect of modulating intermediate formation/decay rates in competitive/ordered model
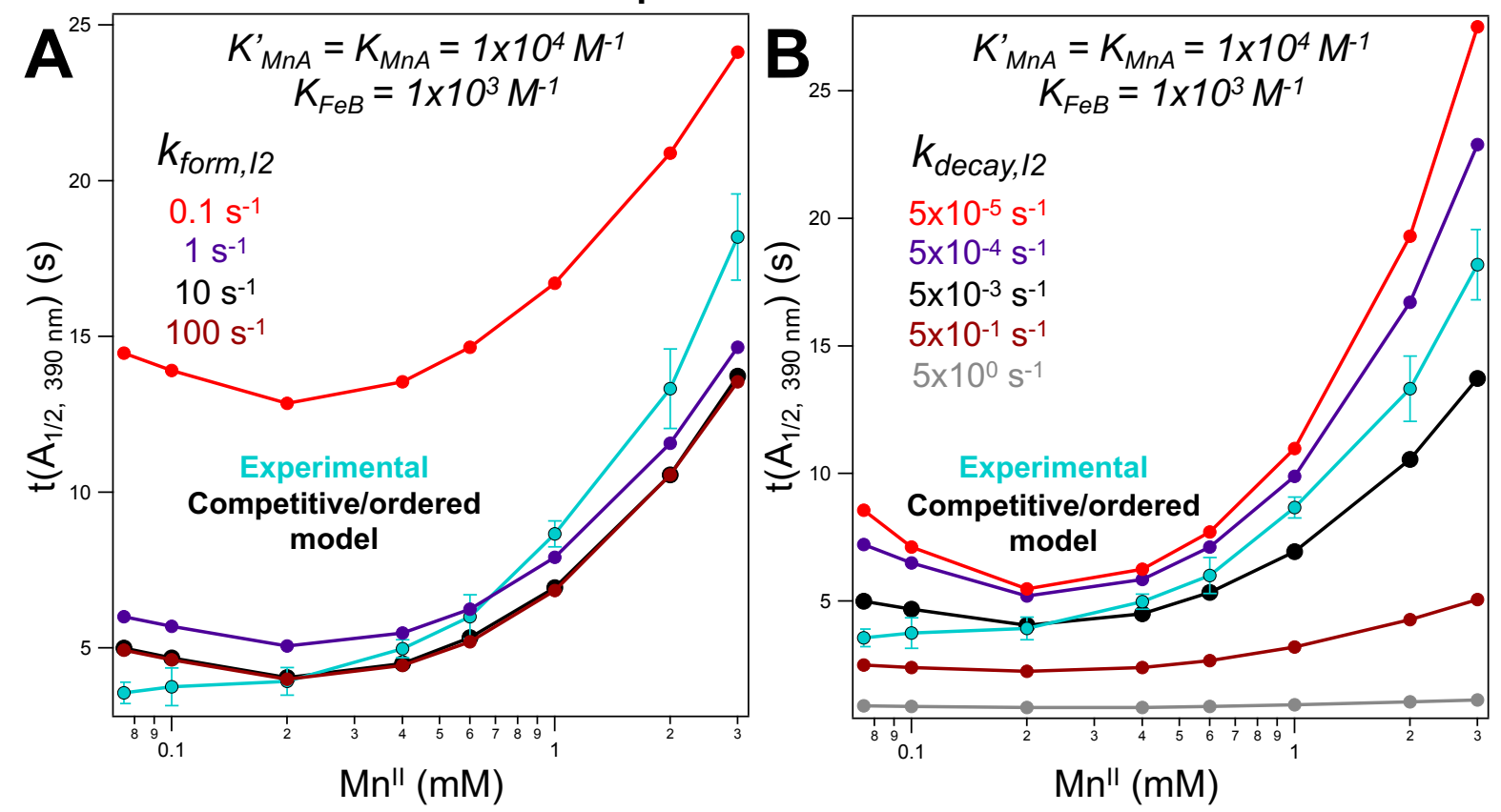

Figure S25. Effect of adjusting rates for $I_{2}$ formation (left) or decay (right) on modeled $t\left(\mathrm{~A}_{1 / 2}\right)$ values using the competitive/ordered model. $I_{2}$ formation was modeled to be slower (red, purple) or faster (dark red, grey) than shown in Figure 4E (black). Experimental data (teal) is shown for comparison. For all metals, $\mathrm{k}_{\text {off }}$ values were held at $10 \mathrm{~s}^{-1}$ and the corresponding $\mathrm{k}_{\mathrm{on}}$ modified. Modified rates of formation or decay (corresponding to $k_{5}$ and $k_{6}$, respectively, as described within the Experimental Section) have divergent effects; slowing the rate of formation results in the same trends at longer $t\left(A_{1 / 2}\right)$ values while increasing the rate of formation by a factor of 10 failed to produce any change in the modeled $t\left(A_{1 / 2}\right)$ values. Slowing the decay rate produces longer $t\left(A_{1 / 2}\right)$ values but also exaggerates the $\mathrm{Mn}^{\mathrm{II}}$ inhibition, while increasing the decay rate results in shorter $\mathrm{t}\left(\mathrm{A}_{1 / 2}\right)$ values and less $\mathrm{Mn}^{\mathrm{II}}$ inhibition. 


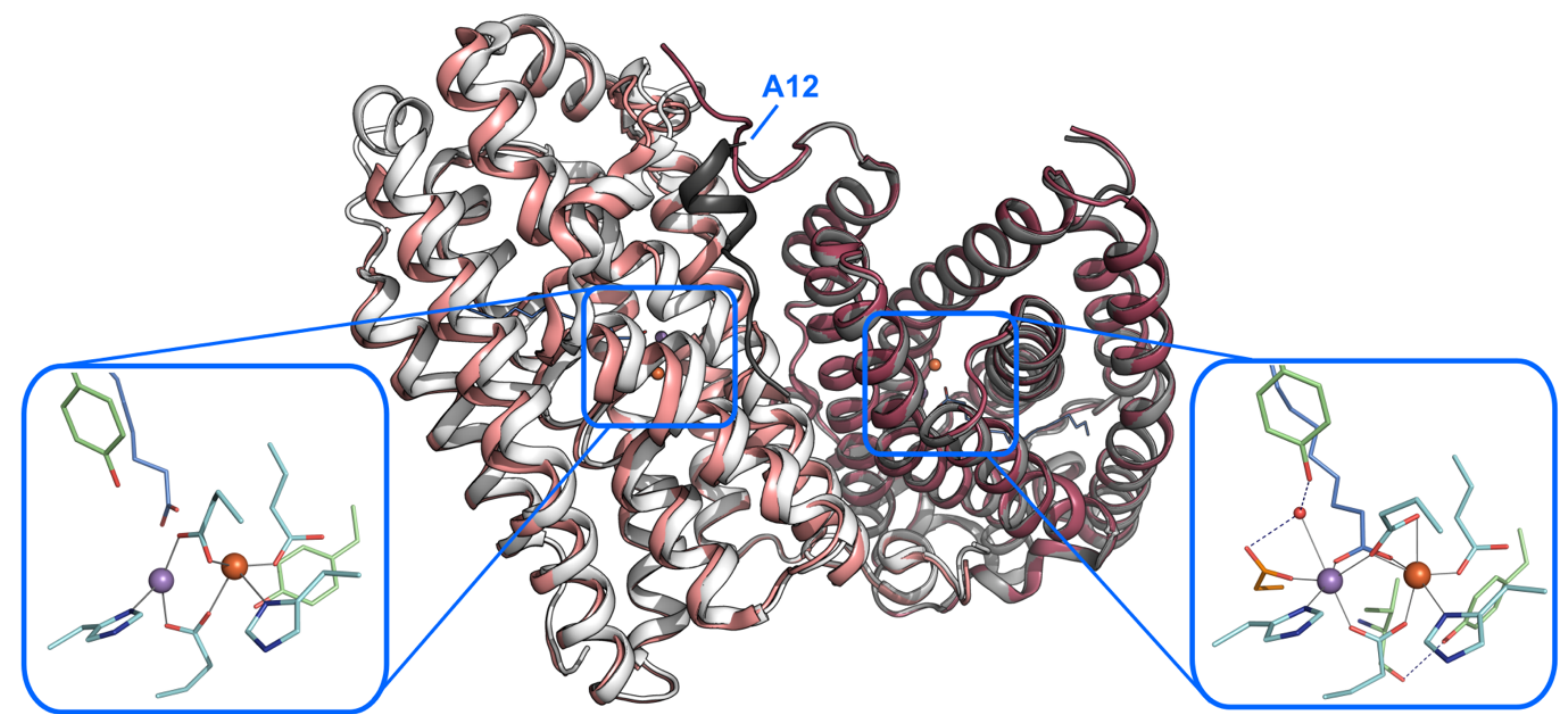

Figure S26. Crystal structure of the E69D R2lox dimer in the reduced state (fully metallated chain A, dark red; partially metallated chain B, salmon), superimposed with the structure of WT R2lox in the same redox state (chain A, grey, N-terminus highlighted in dark grey; chain B, white) (PDB ID 4HR4). ${ }^{2}$ Insets show the active sites of both subunits of E69D R2lox. The N-terminus of the fully metallated subunit of E69D R2lox adopts a different position than that which is observed in WT R2lox. In the WT, the $16 \mathrm{~N}$-terminal residues wrap around the other subunit of the dimer, interacting with helix B, which contains the N-terminal metal ligand E69. ${ }^{2,3}$ In the fully metallated subunit of E69D R2lox, the N-terminus could only be modeled from residue 8 onwards. It also wraps around the other subunit, but in a different direction, lining up with the usual conformation at residue 14. In the largely unmetallated subunit, the N-terminus adopts its usual conformation. These structural differences between the subunits suggest that metal binding/active site ordering and oxygen binding/activation require cooperative action of the two subunits. Interestingly, A12 is a Ramachandran outlier (or very close to being one) in the usual, but not in the unusual Nterminus conformation, suggesting that the high-energy conformation may serve to relay conformational changes between the subunits. 


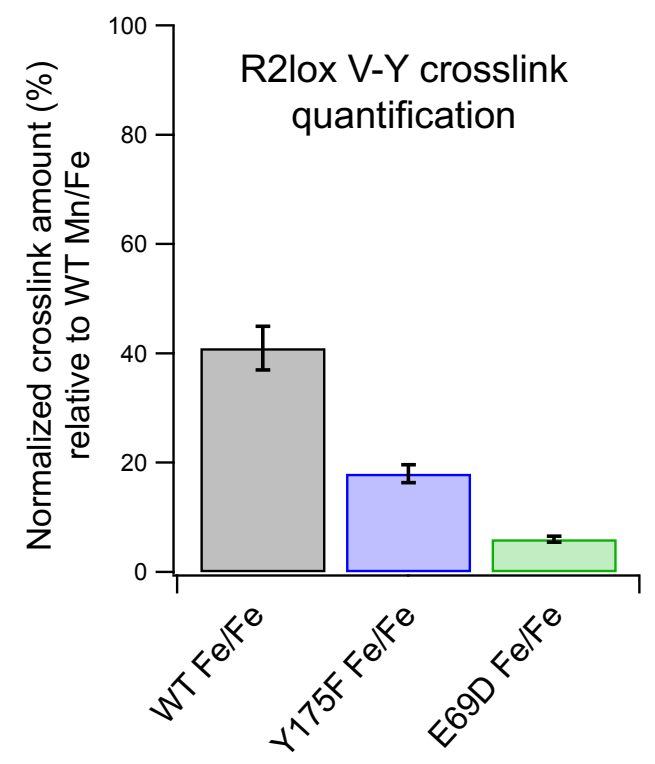

Figure S27. LC-MS analysis of cross-link formation in Fe/Fe WT R2lox compared to Y175F and E69D mutants. Values are from the integrated chromatographic peak areas of the crosslinked peptide AVTLD-AVIRAATVYNMIVE, normalized to the R2lox protein area of the respective LC-MS run. Cross-link levels were normalized to the average of the WT Mn/Fe values. The error bars represent standard error of mean (SEM) across the 9 runs of each sample type (3 biochemical replicates $\mathrm{x} 3$ technical replicates). 


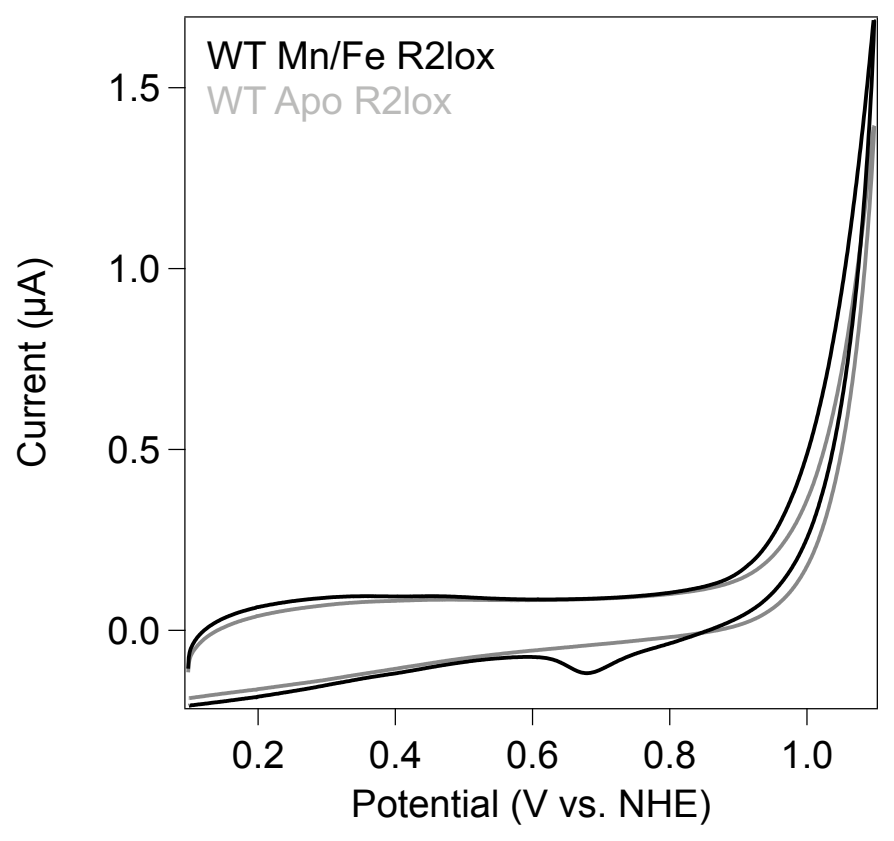

Figure S28. Cyclic voltammograms $(v=10 \mathrm{mV} / \mathrm{s})$ of $100 \mu \mathrm{M} \mathrm{WT} \mathrm{Mn/Fe} \mathrm{(black)} \mathrm{and} \mathrm{apo-R2lox}$ (gray) in $100 \mathrm{mM}$ HEPES, pH 6.0, with $100 \mathrm{mM} \mathrm{NaCl}$. 

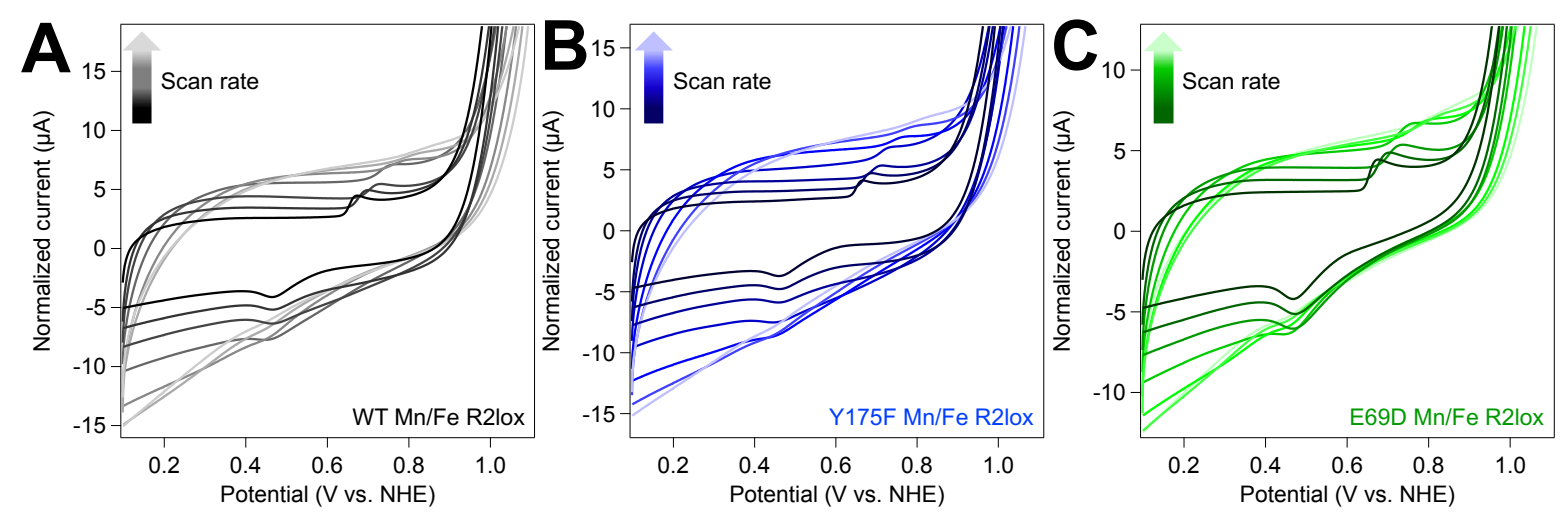

Figure S29. Cyclic voltammograms as a function of scan rate $(v=10 \mathrm{mV} / \mathrm{s}, 25 \mathrm{mV} / \mathrm{s}, 50 \mathrm{mV} / \mathrm{s}$, $100 \mathrm{mV} / \mathrm{s}, 250 \mathrm{mV} / \mathrm{s}, 500 \mathrm{mV} / \mathrm{s}, 1000 \mathrm{mV} / \mathrm{s}$ ) of (A) Mn/Fe WT R2lox (B) Mn/Fe Y175F R2lox and $(\mathbf{C}) \mathrm{Mn} / \mathrm{Fe}$ E69D R2lox. The current was normalized against the square root of the scan rate. All samples contained $100 \mu \mathrm{M}$ protein in $100 \mathrm{mM}$ HEPES with $100 \mathrm{mM} \mathrm{NaCl}, \mathrm{pH} 8.0$ 


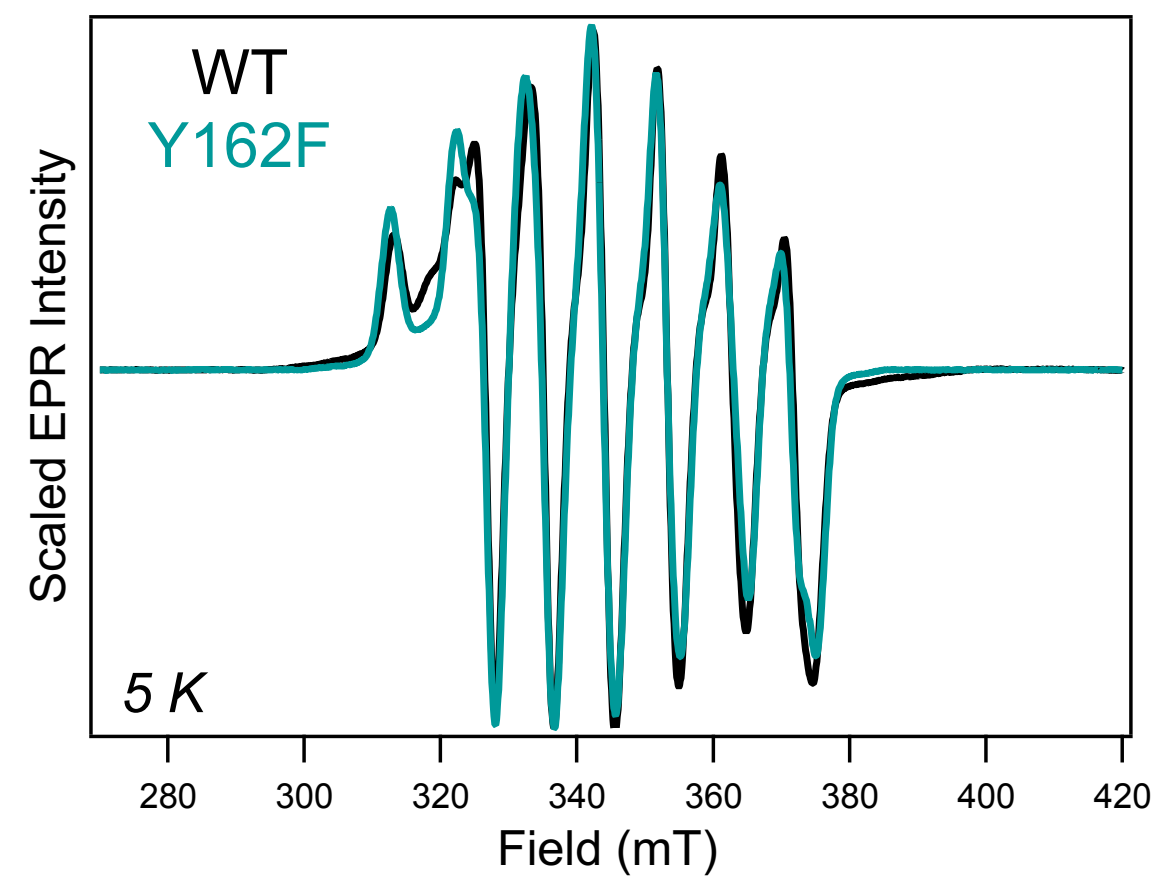

Figure S30. Normalized EPR spectra of Mn/Fe WT compared to that of Y162F R2lox, which does not form the tyrosine-valine crosslink. ${ }^{4}$ 


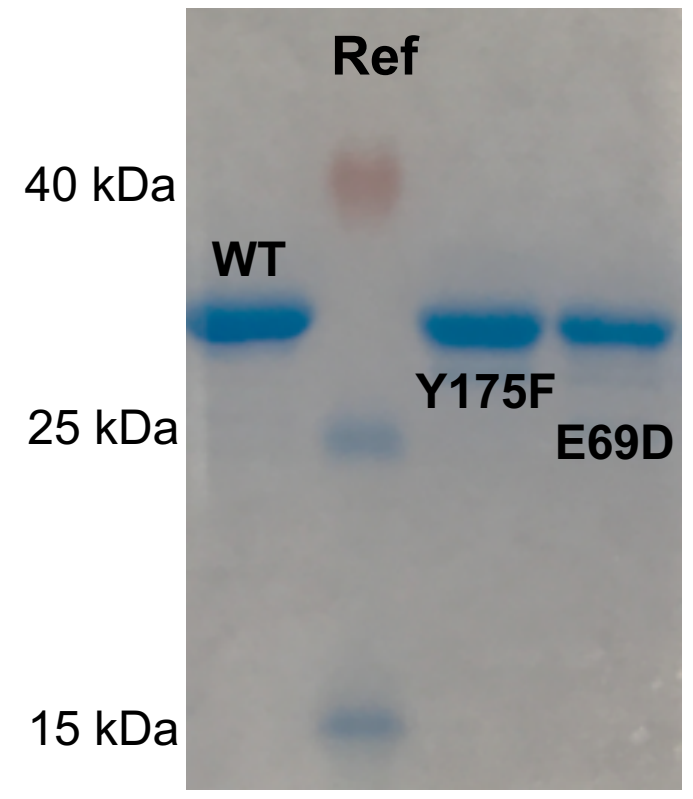

Figure S31. 12\% SDS-PAGE of WT, Y175F, and E69D GkR2loxI referenced against Spectra Multicolor low range protein ladder (Thermo Fisher Scientific). 

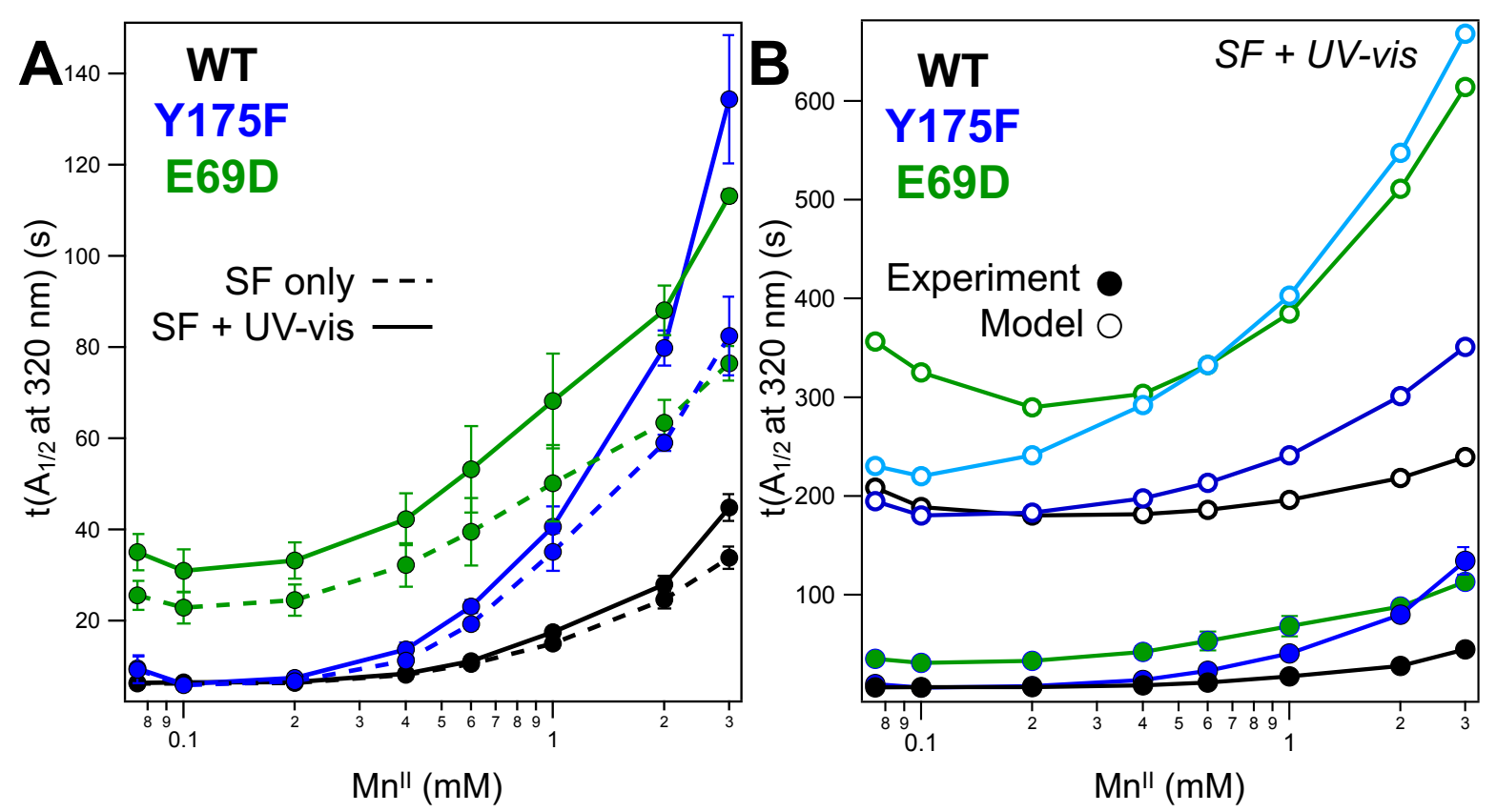

Figure S32. (A) Comparison of $\mathrm{Mn}^{\mathrm{II}}$ inhibition curves generated using either UV-vis $+\mathrm{SF}$ (solid) or SF-only (dashed) datasets. (B) Experimental data from SF + UV-vis overlays are compared to $\mathrm{Mn}^{\mathrm{II}}$ inhibition curves generated from the resting state species modeled out to $3600 \mathrm{~s}$. The modeled data were generated using the competitive/ordered binding model, with $\mathrm{K}_{\mathrm{MnA}}=\mathrm{K}^{\prime}{ }_{\mathrm{MnA}}=10^{4}$ and $\mathrm{K}_{\mathrm{FeB}}=10^{3} ; \mathrm{k}_{\text {off }}$ for all models was set to $10 \mathrm{~s}^{-1}$ and $\mathrm{k}_{\text {on }}$ was adjusted accordingly. 

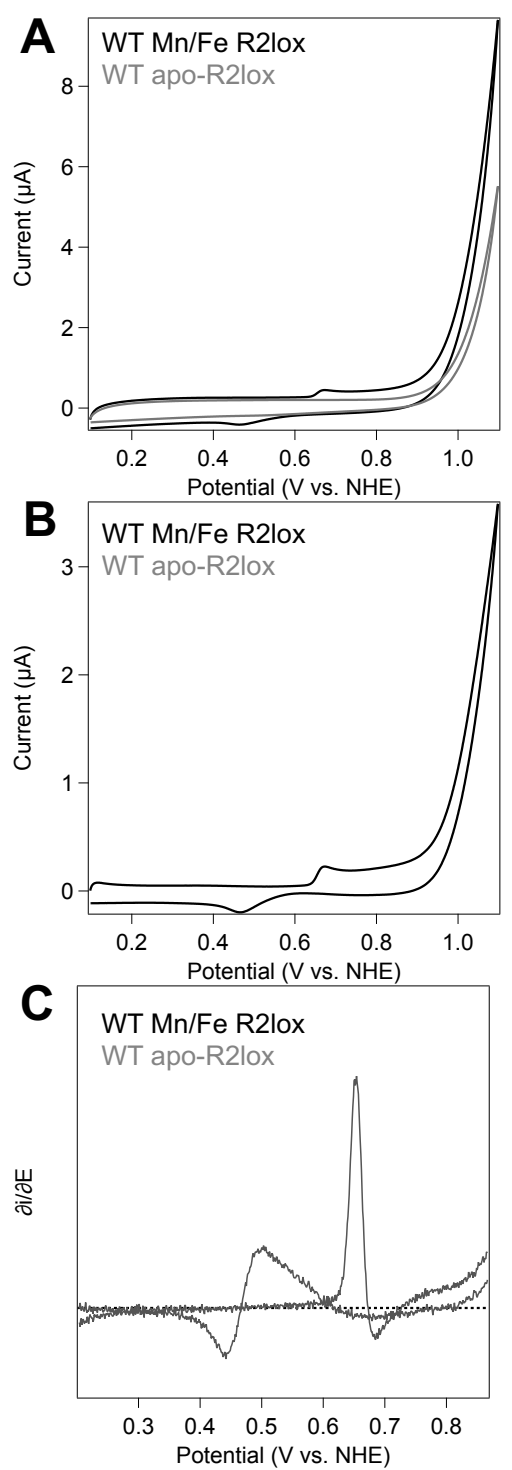

Figure S33. Example electrochemical data workup. (A) Raw cyclic voltammograms of apo- (gray) and WT (black) Mn/Fe R2lox. Cyclic voltammograms $(v=10 \mathrm{mV} / \mathrm{s})$ recorded on $100 \mu \mathrm{M}$ protein in $100 \mathrm{mM}$ HEPES with $100 \mathrm{mM} \mathrm{NaCl}$. (B) Resultant cyclic voltammogram following subtraction of apo-R2lox control as described in the Experimental Section. (C) First derivative of subtracted cyclic voltammogram that was used to determine anodic and cathodic peak potentials. 
Table S1. Spin Hamiltonian parameters from EPR simulations of the resting state species for WT, Y175F, and E69D Mn/Fe R2lox.

\begin{tabular}{|l|l|l|l|}
\hline & $\mathbf{W T}^{\mathrm{a}}$ & $\mathbf{Y 1 7 5 F}$ & E69D \\
\hline $\boldsymbol{g}_{z}$ & 2.034 & 2.032 & 2.036 \\
\hline $\boldsymbol{g}_{\boldsymbol{y}}$ & 1.968 & 1.978 & 1.969 \\
\hline $\boldsymbol{g}_{\boldsymbol{x}}$ & 1.953 & 1.959 & 1.950 \\
\hline $\boldsymbol{A}_{\boldsymbol{z}}{ }^{\boldsymbol{1}}$ & 282 & 266 & 286 \\
\hline $\boldsymbol{A}_{\boldsymbol{y}}$ & 249 & 247 & 253 \\
\hline $\boldsymbol{A}_{\boldsymbol{x}}$ & 257 & 257 & 254 \\
\hline${ }^{\mathbf{5 7}} \mathbf{F e} \boldsymbol{A}_{\boldsymbol{z}}$ & 93.9 & 84.0 & 89.0 \\
\hline${ }^{\mathbf{5 7}} \mathbf{F e} \boldsymbol{A}_{\boldsymbol{y}}$ & 51.6 & 65.0 & 52.0 \\
\hline${ }^{\mathbf{5 7}} \mathbf{F e} \boldsymbol{A}_{\boldsymbol{x}}$ & 62.5 & 65.0 & 62.0 \\
\hline
\end{tabular}

apublished values for WT R2lox. ${ }^{5}$

${ }^{\mathrm{b}}$ Hyperfine (A) values reported in $\mathrm{MHz}$. 
Table S2. Sample calculation of the efficiency of Y175F and E69D R2lox relative to WT towards forming either the $\mathrm{Mn}^{\mathrm{III}}(\mu-\mathrm{OH}) \mathrm{Fe}^{\mathrm{III}}$ cofactor or the cross-linked peptide.

\begin{tabular}{|c|c|c|c|}
\hline & & $\%$ Efficiency $^{\mathbf{a}}$ & [Conc.] $(\mu \mathrm{M})^{\mathrm{b}}$ \\
\hline \multirow{3}{*}{ WT } & Apo-R2lox & -- & $100 \mu \mathrm{M}$ \\
\hline & $\mathrm{Mn}^{\mathrm{III}}(\mu-\mathrm{OH}) \mathrm{Fe}^{\mathrm{III}}(\mathrm{EPR})$ & -- & $40 \mu \mathrm{M}$ \\
\hline & Cross-link (M/S) & -- & $20 \mu \mathrm{M}^{\mathrm{c}}$ \\
\hline \multirow{3}{*}{ Y175F } & Apo-R2lox & -- & $100 \mu \mathrm{M}$ \\
\hline & $\mathrm{Mn}^{\mathrm{III}}(\mu-\mathrm{OH}) \mathrm{Fe}^{\mathrm{III}}(\mathrm{EPR})$ & $110 \%$ & $44 \mu \mathrm{M}$ \\
\hline & Cross-link (M/S) & $80 \%$ & $16 \mu \mathrm{M}$ \\
\hline \multirow{3}{*}{ E69D } & Apo-R2lox & -- & $100 \mu \mathrm{M}$ \\
\hline & $\mathrm{Mn}^{\mathrm{III}}(\mu-\mathrm{OH}) \mathrm{Fe}^{\mathrm{III}}(\mathrm{EPR})$ & $20 \%$ & $8 \mu \mathrm{M}$ \\
\hline & Cross-link (M/S) & $40 \%$ & $8 \mu \mathrm{M}$ \\
\hline
\end{tabular}

${ }^{a}$ Values obtained from data shown in Figure 8 are given with respect to WT

${ }^{b}$ Absolute concentrations for each mutant are calculated by applying the percent efficiency to WT values. A sample concentration of $100 \mu \mathrm{M}$ apo-protein was used for illustrative purposes. ${ }^{\mathrm{c}}$ An initial assumption of 50\% efficiency was made regarding cross-link formation for the $\mathrm{Mn}^{\mathrm{III}}(\mu-\mathrm{OH}) \mathrm{Fe}^{\mathrm{III}}$ cofactor in WT R2lox 
Table S3. Crystallographic data statistics

\begin{tabular}{|l|c|c|c|c|}
\hline R2lox mutant & E69D & E69D & Y175F & Y175F \\
\hline Soaking condition & anoxic Mn+Fe & aerobic Mn+Fe & anoxic Mn+Fe & aerobic Mn+Fe \\
\hline Beamline & X06SA/SLS & X06SA/SLS & X06SA/SLS & X06SA/SLS \\
Detector & Pilatus 6MF & Pilatus 6M & Pilatus 6MF & Pilatus 6M \\
Wavelength $(\AA)$ & 1.00 & 1.00 & 1.00 & 1.00 \\
Resolution range $(\AA)$ & $50.00-2.09$ & $50.00-1.90$ & $50.00-1.60$ & $50.00-1.75$ \\
Space group & $(2.22-2.09)$ & $(2.02-1.90)$ & $(1.69-1.60)$ & $(1.86-1.75)$ \\
Unit cell dimensions & $160.81,55.96$, & $55.87,96.82$, & $129.12,97.79$, & $55.87,97.30$, \\
a, b, c $(\AA)$ & 98.06 & 129.20 & 55.99 & 129.11 \\
$\beta\left({ }^{\circ}\right)$ & 126.23 & 90.00 & 90.00 & 90.00 \\
Unique reflections & $40539(6205)$ & $27535(4310)$ & $94346(14804)$ & $35227(5598)$ \\
Multiplicity & 3.4 & 3.3 & 6.5 & 4.1 \\
Completeness $(\%)$ & $96.3(91.7)$ & $98.8(97.1)$ & $99.4(97.6)$ & $98.7(98.2)$ \\
I/ $\sigma(\mathrm{I})$ & $13.00(1.65)$ & $14.49(1.79)$ & $15.29(1.97)$ & $19.90(1.74)$ \\
$R_{\text {merge }}(\%)$ & $6.7(79.9)$ & $5.0(65.8)$ & $6.4(80.5)$ & $3.5(76.0)$ \\
$R_{\text {meas }}(\%)$ & $7.9(94.5)$ & $6.0(79.0)$ & $7.0(87.8)$ & $4.0(87.6)$ \\
CC $1 / 2{ }^{\text {a }}$ & $99.9(72.3)$ & $99.9(69.1)$ & $99.8(73.5)$ & $99.9(68.4)$ \\
\hline
\end{tabular}

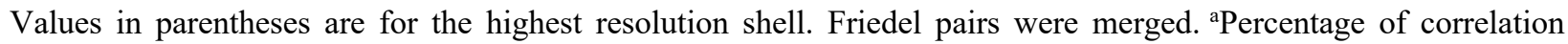
between intensities from random half-datasets. ${ }^{6}$ The correlation is significant at the $0.1 \%$ level in all resolution shells in all datasets. 
Table S4. Refinement statistics

\begin{tabular}{|c|c|c|c|c|}
\hline \multirow{2}{*}{$\frac{\text { R2lox mutant }}{\text { Soaking condition }}$} & \multicolumn{2}{|c|}{ E69D } & \multicolumn{2}{|c|}{ Y175F } \\
\hline & anoxic $\mathrm{Mn}+\mathrm{Fe}$ & aerobic $\mathrm{Mn}+\mathbf{F e}$ & $\operatorname{anoxic} \mathrm{Mn}+\mathbf{F e}$ & aerobic $\mathrm{Mn}+\mathbf{F e}$ \\
\hline PDB ID & 6QK0 & 6QJV & 6QK2 & 6QK1 \\
\hline Resolution range ( $(\AA)$ & $48.60-2.09$ & $39.35-1.91$ & $48.60-1.60$ & $38.85-1.75$ \\
\hline Reflections used & 40492 & 27517 & 94335 & 35224 \\
\hline$R_{\text {work }} / R_{\text {free }}(\%)^{\mathrm{a}}$ & $20.9 / 26.3$ & $16.4 / 20.4$ & $17.3 / 19.0$ & $17.9 / 22.1$ \\
\hline Coordinate error $(\AA)$ & 0.33 & 0.22 & 0.16 & 0.23 \\
\hline Non-H atoms & 4653 & 2497 & 5068 & 2511 \\
\hline Protein residues ${ }^{\mathrm{b}}$ & $\begin{array}{l}547(8-287 / 4- \\
251 ; 263-286)\end{array}$ & $285(2-286)$ & $\begin{array}{c}570(2-286 / 2- \\
286)\end{array}$ & $285(2-286)$ \\
\hline Water molecules & 82 & 112 & 304 & 118 \\
\hline Ligand molecules & 2 & 1 & 2 & 1 \\
\hline Metal ions & 6 & 3 & 6 & 3 \\
\hline $\operatorname{rmsd}$ bonds $(\AA)^{\mathrm{c}}$ & 0.021 & 0.016 & 0.015 & 0.018 \\
\hline rmsd angles $\left({ }^{\circ}\right)^{\mathrm{c}}$ & 1.262 & 1.023 & 1.052 & 1.086 \\
\hline $\begin{array}{l}\text { Ramachandran favored/allowed/ } \\
\text { outliers }(\%)^{\mathrm{d}}\end{array}$ & $97.0 / 2.8 / 0.2$ & $97.2 / 2.4 / 0.4$ & $98.2 / 1.4 / 0.4$ & $97.2 / 2.4 / 0.4$ \\
\hline Clashscore $^{\mathrm{d}}$ & 4.75 & 2.33 & 2.76 & 2.32 \\
\hline Wilson $B$ factor $\left(\AA^{2}\right)$ & 39.9 & 30.6 & 23.4 & 31.2 \\
\hline Average $B$ factors $\left(\AA^{2}\right)^{\mathrm{e}, \mathrm{f}}$ & & & & \\
\hline all atoms & 57.2 & 41.5 & 35.1 & 44.4 \\
\hline protein main and side chains & $56.9 / 57.5$ & 41.4 & $35.6 / 34.3$ & 44.4 \\
\hline site 1 metal ion & $38.7 / 62.0$ & 23.8 & $18.4 / 18.1$ & 26.6 \\
\hline site 2 metal ion & $45.1 / 44.7$ & 26.8 & $22.6 / 22.8$ & 32.5 \\
\hline additional metal ions & $78.2 / 76.8$ & 79.7 & $37.7 / 37.7$ & 76.4 \\
\hline ligand & $54.2 / 71.4$ & 44.4 & $42.4 / 40.5$ & 48.6 \\
\hline water & 47.5 & 42.6 & 38.1 & 44.3 \\
\hline Occupancies $<1.0^{\mathrm{f}}$ & & & & \\
\hline site 1 metal ion & $1.00 / 0.93$ & - & - & - \\
\hline site 2 metal ion & $1.00 / 0.61$ & - & - & - \\
\hline additional metal ions & $1.00 / 0.99$ & 0.95 & $0.66 / 0.69$ & 0.90 \\
\hline
\end{tabular}

${ }^{\mathrm{a}} R_{\text {free }}$ is calculated from a randomly selected subset of approximately 2000 reflections (corresponding to up to $5 \%$ of reflections) excluded from refinement. ${ }^{b}$ Residues out of the 302 residue full-length protein included in the final model are given in parentheses. ${ }^{c}$ Root-mean-square deviation from ideal geometry. ${ }^{\mathrm{d}}$ Geometry statistics were calculated with MolProbity. ${ }^{7}$ eAverage $B$ factors were calculated with Baverage in the CCP4 suit. ${ }^{8}$ Where there are 2 molecules in the asymmetric unit, the values for chain $A$ and chain $B$ are given separately where applicable (A/B). 


\section{References}

(1) Kutin, Y.; Srinivas, V.; Fritz, M.; Kositzki, R.; Shafaat, H. S.; Birrell, J.; Bill, E.; Haumann, M.; Lubitz, W.; Högbom, M.; Griese, J.J.; Cox, N. Divergent Assembly Mechanisms of the Manganese/Iron Cofactors in R2lox and R2c Proteins. J. Inorg. Biochem. 2016, 162, 164177.

(2) Griese, J. J.; Roos, K.; Cox, N.; Shafaat, H. S.; Branca, R. M. M.; Lehtiö, J.; Gräslund, A.; Lubitz, W.; Siegbahn, P. E. M.; Högbom, M. Direct Observation of Structurally Encoded Metal Discrimination and Ether Bond Formation in a Heterodinuclear Metalloprotein. Proc. Natl. Acad. Sci. 2013, 110 (43), 17189-17194.

(3) Griese, J. J.; Kositzki, R.; Schrapers, P.; Branca, R. M. M.; Nordström, A.; Lehtiö, J.; Haumann, M.; Högbom, M. Structural Basis for Oxygen Activation at a Heterodinuclear Manganese/Iron Cofactor. J. Biol. Chem. 2015, 290 (42), 25254-25272.

(4) Maugeri, P. T.; Griese, J. J.; Branca, R. M.; Miller, E. K.; Smith, Z. R.; Eirich, J.; Högbom, M.; Shafaat, H. S. Driving Protein Conformational Changes with Light: Photoinduced Structural Rearrangement in a Heterobimetallic Oxidase. J. Am. Chem. Soc. 2018, 140 (4), 1471-1480.

(5) Shafaat, H. S.; Griese, J. J.; Pantazis, D. A.; Roos, K.; Andersson, C. S.; Popović-Bijelić, A.; Gräslund, A.; Siegbahn, P. E. M.; Neese, F.; Lubitz, W.; Högbom, M.; Cox, N. Electronic Structural Flexibility of Heterobimetallic Mn/Fe Cofactors: R2lox and R2c Proteins. J. Am. Chem. Soc. 2014, 136 (38), 13399-13409.

(6) Karplus, P. A.; Diederichs, K. Linking Crystallographic Model and Data Quality. Science 2012, 336 (6084), 1030-1033.

(7) Chen, V. B.; Arendall, W. B.; Headd, J. J.; Keedy, D. A.; Immormino, R. M.; Kapral, G. J.; Murray, L. W.; Richardson, J. S.; Richardson, D. C. MolProbity: All-Atom Structure Validation for Macromolecular Crystallography. Acta Crystallogr. D Biol. Crystallogr. 2010, 66 (1), 12-21.

(8) Winn, M. D.; Ballard, C. C.; Cowtan, K. D.; Dodson, E. J.; Emsley, P.; Evans, P. R.; Keegan, R. M.; Krissinel, E. B.; Leslie, A. G. W.; McCoy, A.; McNicholas, S.J.; Murshudov, G.N.; Pannu, N.S.; Potterton, E.A.; Powell, H.R.; Read, R.J.; Vagin, A.; Wilson, K.S. Overview of the CCP4 Suite and Current Developments. Acta Crystallogr. D Biol. Crystallogr. 2011, 67 (4), 235-242. 